\title{
Simple kinematic models for the environmental interaction of tropical cyclones in vertical wind shear
}

\author{
M. Riemer ${ }^{1, *}$ and M. T. Montgomery ${ }^{1,2}$ \\ ${ }^{1}$ Department of Meteorology, Naval Postgraduate School, Monterey, CA, USA \\ ${ }^{2}$ NOAA's Hurricane Research Division, Miami, FL, USA \\ * current address: Institut für Physik der Atmosphäre, Johannes Gutenberg-Universität, Mainz, Germany
}

Received: 28 August 2010 - Published in Atmos. Chem. Phys. Discuss.: 16 November 2010

Revised: 23 May 2011 - Accepted: 19 August 2011 - Published: 12 September 2011

\begin{abstract}
A major impediment to the intensity forecast of tropical cyclones (TCs) is believed to be associated with the interaction of TCs with dry environmental air. However, the conditions under which pronounced TC-environment interaction takes place are not well understood. As a step towards improving our understanding of this problem, we analyze here the flow topology of a TC immersed in an environment of vertical wind shear in an idealized, three-dimensional, convection-permitting numerical experiment. A set of distinct streamlines, the so-called manifolds, can be identified under the assumptions of steady and layer-wise horizontal flow. The manifolds are shown to divide the flow around the TC into distinct regions.

The manifold structure in our numerical experiment is more complex than the well-known manifold structure of a non-divergent point vortex in uniform background flow. In particular, one manifold spirals inwards and ends in a limit cycle, a meso-scale dividing streamline encompassing the eyewall above the layer of strong inflow associated with surface friction and below the outflow layer in the upper troposphere. From the perspective of a steady and layer-wise horizontal flow model, the eyewall is well protected from the intrusion of environmental air. In order for the environmental air to intrude into the inner-core convection, time-dependent and/or vertical motions, which are prevalent in the TC innercore, are necessary. Air with the highest values of moistentropy resides within the limit cycle. This "moist envelope" is distorted considerably by the imposed vertical wind shear, and the shape of the moist envelope is closely related to the shape of the limit cycle. In a first approximation, the distribution of high- and low- $\theta_{e}$ air around the TC at low to midlevels is governed by the stirring of convectively modified air by the steady, horizontal flow.
\end{abstract}

\section{Correspondence to: M. Riemer} (mriemer@uni-mainz.de)
Motivated by the results from the idealized numerical experiment, an analogue model based on a weakly divergent point vortex in background flow is formulated. The simple kinematic model captures the essence of many salient features of the manifold structure in the numerical experiment. A regime diagram representing realistic values of TC intensity and vertical wind shear can be constructed for the pointvortex model. The results indicate distinct scenarios of environmental interaction depending on the ratio of storm intensity and vertical-shear magnitude. Further implications of the new results derived from the manifold analysis for TCs in the real atmosphere are discussed.

\section{Tropical cyclone intensity and environmental air interaction}

It has long been recognized that the interaction of tropical cyclones (TCs) with environmental air can have a detrimental effect on storm intensity. The interaction can be expected to be particularly pronounced when vertical shear of the environmental horizontal wind is present. Vertical wind shear ensures that at some height levels the environmental flow is distinct from the motion of the storm and considerable storm-relative environmental flow arises at such levels (e.g. Willoughby et al., 1984; Marks et al., 1992; Bender, 1997). This storm-relative flow then advects environmental air towards the TC. If low moist-entropy (or equivalent potential temperature, $\theta_{e}$ ) air associated with the environment can intrude into the eyewall updrafts, then the conversion of heat into kinetic energy within the TC's power machine is frustrated and the storm can be expected to weaken (Tang and Emanuel, 2010a). Moistening and warming of the environmental air before reaching the eyewall updrafts may diminish this detrimental impact on TC intensity.

Simpson and Riehl (1958) were the first to propose that storm-relative environmental flow "may act as a constraint

Published by Copernicus Publications on behalf of the European Geosciences Union. 
upon the hurricane heat engine". Based on an inspection of the storm-relative radial flow at mid-levels (their Fig. 4), they noted "a prominent movement of environmental air through the storm" and suggested that "the vortex was ventilated by invading colder masses of air". This ventilation of the TC vortex by storm-relative environmental flow is widely invoked to explain the intensity evolution of observed storms (e.g. Willoughby et al., 1984; Marks et al., 1992; Shelton and Molinari, 2009). Emanuel et al. (2004) developed an empirical parameterization within an axisymmetric model framework that attempts to account for the ventilation of low- $\theta_{e}$ air through the TC core at mid-levels in association with an imposed vertical wind shear ${ }^{1}$. A somewhat related pathway of environmental interaction in vertical shear was proposed by Frank and Ritchie (2001). In their model, shear-induced eddy fluxes of potential temperature were hypothesized to erode the upper-level warm core, leading to an increase of the minimum surface pressure by hydrostatic arguments, and ultimately to a decrease of TC intensity.

When considering TC-environment interaction it needs to be borne in mind that a mature TC constitutes a strong atmospheric vortex. This is particularly true when trying to draw conclusions based solely on the storm-relative asymmetric flow, i.e. the storm-relative flow that results after subtracting the axisymmetric TC circulation. The asymmetric flow may serve as an approximation for the environmental flow. The storm-relative asymmetric flow has been referred to as "cross-vortex flow" (Willoughby et al., 1984; Black et al., 2002) or there has been the tacit assumption that air flows through the inner core (Simpson and Riehl, 1958; Bender, 1997; Zhang and Kieu, 2006). The strong swirling winds, however, act to significantly deflect the storm-relative asymmetric flow ${ }^{2}$. Air parcel trajectories in a mature TC, of course, differ greatly from the streamlines of the stormrelative asymmetric flow. This distinction does not always seem to be clear in a number of previous studies. Only for relatively weak storms and strong storm-relative environmental flow does it seem plausible that environmental air will actually penetrate the eyewall of a TC (e.g. Hurricane Claudette (2003), Shelton and Molinari, 2009). The focus of this paper is to explore the limitations of vortex-environment interaction based on the above kinematic considerations.

The foregoing ideas hint that a more efficient way to ingest dry environmental air into the eyewall updrafts is through the storm's inflow layer. In the current study, the term "inflow layer" will refer to the layer of strong inflow associated

\footnotetext{
${ }^{1}$ This empirical parameterization is now used each hurricane season in the CHIPS forecast model of Kerry Emanuel.

${ }^{2}$ The strong radial shear of the swirling winds tends also to damp any asymmetry that tries to invade the TC. This is the so-called vortex axisymmetrization process (e.g. Melander et al., 1987; Carr and Williams, 1989) that is a well-known essential ingredient in the robustness and persistence of coherent vortex structures in quasi two-dimensional flows. The axisymmetrization process will not be considered explicitly in this study.
}

with surface friction in the lowest $1 \mathrm{~km}-1.5 \mathrm{~km}$ where the departure from gradient wind balance in the radial direction is generally a maximum (e.g., Bui et al. (2009), Figs. 5 and 6 and accompanying discussion). Riemer et al. (2010, RMN hereafter) have shown how the interaction of vertical shear with a mature TC can form persistent, vortex-scale downdrafts which flush the inflow layer with low- $\theta_{e}$ air and effectively reduce eyewall $\theta_{e}$ values by $3 \mathrm{~K}$ to $9 \mathrm{~K}$, depending on the magnitude of vertical shear. Without explicit consideration of vertical shear effects, earlier studies have shown that downdrafts associated with individual rain bands may bring low- $\theta_{e}$ air into the inflow layer (Barnes et al., 1983; Powell, 1990). Powell has estimated that the replenishment of this air by surface fluxes may not be complete before reaching the eyewall and concluded that this process could affect the intensity evolution of the storm. In a more recent modeling study, Kimball (2006) has found that "dry environmental air above approximately $850 \mathrm{hPa}$ rotates cyclonically and inward around the storm center" and may be subsequently involved in downdrafts close to the eyewall. Two schematic diagrams summarizing how the TC power-machine may be frustrated by mid-level ventilation and pronounced downdrafts are given in Figs. 1 and 2 of RMN.

The processes discussed above, the ventilation of eyewall convection, the erosion of the upper-level warm core, and the depression of inflow layer $\theta_{e}$ by downdrafts, are widely believed to operate in real storms ${ }^{3}$. The relative importance of these processes for intensity modification is generally unknown and the environmental conditions under which they may operate are not well understood. This is consistent with the fact that the interaction with vertical shear and/or the presence of dry air in the vicinity of a storm poses an enhanced forecast challenge. Dry air can be observed to impinge on TCs (e.g. Zipser et al., 2009, tropical storm Debby), yet it has not been entirely clear how storm intensity is affected. Idealized experiments demonstrate that dry air may wrap all around a developing TC in quiescent environment without affecting storm intensity at all (S. A. Braun, personal communication, 2010). Similar questions arise for TCs that interact with the Saharan air layer (SAL) ${ }^{4}$.

Aspects of TC-environment interaction have received considerable attention on the convective scale and the scale of individual rain bands (e.g. The Hurricane Rainband and Intensity Change Experiment, Houze et al., 2006). However, we believe that an overarching framework for this problem is still lacking. The goal of the present study is to contribute

\footnotetext{
${ }^{3} \mathrm{~A}$ kinematic impact on $\mathrm{TC}$ intensity for a sheared storm arises through vortex tilt, i.e. the vertical misalignment of the TC's potential vorticity tower. However, as discussed in the introduction of $\mathrm{RMN}$, there is strong indication that this kinematic effect is not a primary intensity change mechanism.

${ }^{4}$ The effect of SAL, however, is thought to be more complex than simply providing a source of very dry air (e.g. Dunion and Velden, 2004).
} 
to such a framework by taking a broader-scale viewpoint and considering the larger-scale air mass distribution around a TC.

\subsection{Revisiting a horizontal and steady flow model}

For steady, two-dimensional flow, the flow topology can be examined by analyzing the hyperbolic fixed points and their associated manifolds. At a fixed point, both horizontal wind components vanish. A fixed point is hyperbolic when air parcels in the vicinity of this point are stretched in one direction and contracted in another direction. Mathematically speaking, when the flow field is linearized around a hyperbolic fixed point, the eigenvalues of the strain tensor are real and of opposite sign. Here, we will refer to hyperbolic fixed points simply as stagnation points. Streamlines that emanate from a stagnation point constitute the hyperbolic manifolds of the flow. A manifold along which the flow approaches the stagnation point is called a stable manifold. A manifold along which the flow departs from the stagnation point is called an unstable manifold. Hyperbolic manifolds divide the flow into different regimes and thus determine the topology of the flow. Note that the identification of stagnation points is crucial for the objective analysis of the flow topology.

For steady, non-divergent flow the interaction of a vortex with the background flow gives rise to a manifold that separates the area of rotation-dominated flow from the largerscale environment. For this special case, we refer to the manifold as a dividing streamline. Inside of the dividing streamline, all streamlines are closed and air parcels are confined to the vortex. Outside the dividing streamline, the flow passes around the vortex. The stagnation point in this example is found where the velocity field of the vortex and that of the background flow cancel.

Based on this basic fluid-dynamical concept, Willoughby et al. (1984, hereafter WMF84) proposed that for a TC in storm-relative environmental flow "the streamline pattern is divided into a vortex core where the streamlines form a closed gyre and the vortex envelope where they curve around the core in a wavelike pattern" (Fig. 1). These authors noted further that "in the closed gyre, the air moves with the vortex; in the envelope, environmental air passes through the vortex and around the core". Willoughby et al. noted clearly the existence and importance of flow boundaries in the vicinity of a vertically sheared TC. These authors indicated also that the air within the closed gyre has generally higher $\theta_{e}$ values than outside of the gyre.

WMF84's seminal hypothesis of separated flow regions with distinct thermodynamic properties and its ramifications for the interaction of TCs with environmental air have received little attention in the literature. WMF84's results are derived from a low wave-number analysis of observational data from a number of flight legs within $150 \mathrm{~km}$ of the storm center. Most notably, the stagnation point was not contained within the domain considered by WMF84. We believe that

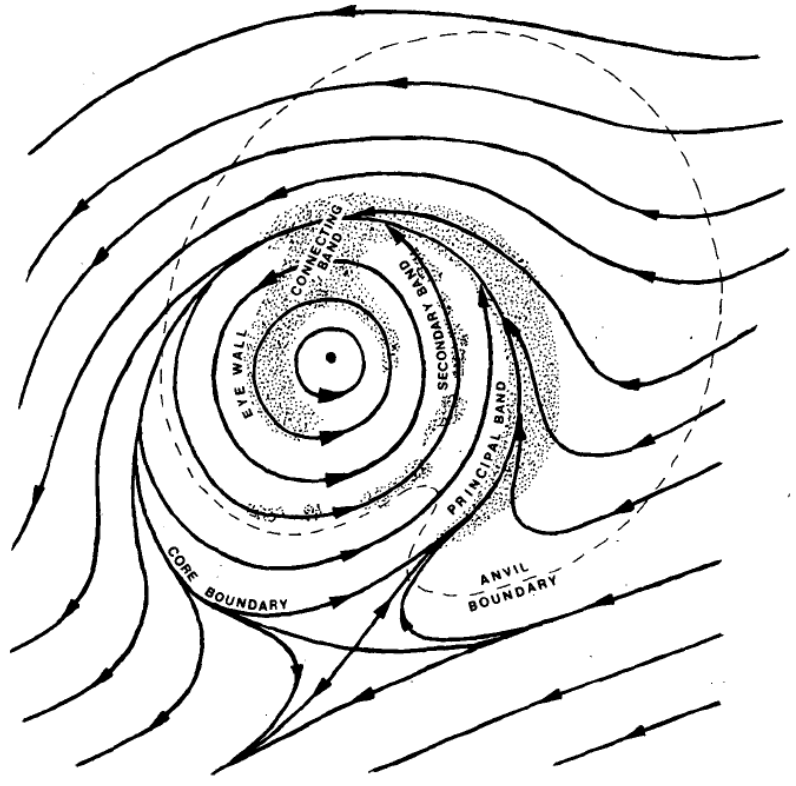

Fig. 1. Willoughby et al.'s hypothesized flow topology of a mature TC in northeasterly storm-relative flow above the inflow layer (approx. $850 \mathrm{hPa}$, their Fig. 18). The closed gyre around the center is supposed to contain the moist "vortex air" while drier environmental air is found outside the dividing streamline. The shading indicates regions of deep convection. The principal, secondary, and connecting band constitute the so-called stationary band complex.

it is worthwhile to revisit WMF84's seminal hypothesis and analyze the flow topology of a TC in vertical wind shear with a much more complete data set from a recent idealized numerical experiment.

Consistent with WMF84's analysis, we will assume that the environmental flow is layer-wise horizontal and steady. The first assumption is supported by scaling arguments for large-scale tropical circulations (Charney, 1963; Holton, 2004 , Ch. 11) and is a good approximation outside of convective regions. The time scale of significant intensity modification during vertical shear interaction in our numerical experiment is consistent with TCs in the real atmosphere (e.g. Zehr, 2003). During the first $24 \mathrm{~h}$ after shear is imposed, the $\mathrm{TC}$ in the experiment both weakens and then re-intensifies by approx. $10 \mathrm{~ms}^{-1}$. The initial vertical shear flow that is imposed on the TC is found to be steady in the environment. Although axisymmetrization of the weak potential vorticity gradient associated with the environmental flow may lead to some modification of this flow in the vicinity of the TC, we show in Sect. 2.1.3 that the flow topology remains essentially the same over the 1-day time period under consideration. Barring interaction with an upper-level trough or a strong jet stream, e.g. as during extratropical transition, changes of the large-scale flow in which TCs are embedded in the real atmosphere are usually modest on the time scale of 1 day 
also. Thus, we believe that the steady-flow approximation provides scientific value for studying TC-environment interaction in the real atmosphere.

In the vicinity of the eyewall, however, the assumptions of steady and layer-wise horizontal flow break down. In a mature TC, vortex Rossby waves (VRWs) and their coupling to convection and the boundary layer are arguably the most important transient flow features in this region (Montgomery and Kallenbach, 1997; Chen and Yau, 2001; Wang, 2002; Chen et al., 2003). For an intensifying TC, rotating deep convective structures, also referred to as "vortical hot towers", can be expected to be dominant features (Nguyen et al., 2008). This rich fluid dynamical behavior is not considered here. An extensive trajectory analysis of the timedependent, three-dimensional flow to quantify the mid-level ventilation of eyewall convection in a numerical simulation of Hurricane Bonnie (1998) has been performed by Cram et al. (2007). The analyses therein were limited to a $5 \mathrm{~h}$ timescale for back trajectories and confined to a $100 \mathrm{~km}$ radius from the center of the vortex. That study also did not consider the broader-scale interactions between the environment and the inner-core region.

A natural extension of the current study is to examine the Lagrangian coherent structures (LCS) that govern the interaction of fluid from distinct regions in the unsteady, threedimensional flow (e.g. Haller, 2001). These LCS are the finite-time manifolds of the time-dependent flow. First analyses of such LCS in TCs have recently been performed by Sapsis and Haller (2009) and Rutherford et al. (2010). We anticipate that the analysis of LCS in a vertically sheared TC will yield further insight into the interaction of the TC with environmental air. The current study nonetheless should provide a useful framework for interpreting the results from an analysis of the LCS.

An outline for the paper is as follows. In Sect. 2 we use the idealized numerical experiment of RMN to illustrate the flow topology and its relation to distinct air masses around the vertically sheared TC. Motivated by the model results we then formulate an analogue model based on a point vortex with singular mass sink in Sect. 3. The analogue model is shown to capture the basic features of the flow topology in the idealized numerical experiment. Section 4 considers application of the analogue model to the problem of TC-environment interaction in the real atmosphere. Conclusions are presented in Sect. 5.

\section{Flow topology in an idealized numerical experiment of TC-shear interaction}

We analyze the flow topology of a mature TC in an idealized numerical experiment. The experiment analyzed herein is the 15 mps case of RMN. The Regional Atmospheric Modeling System (RAMS, Pielke et al., 1992; Cotton et al., 2003) was used to perform this experiment. We have deliberately employed a very simple set of parametrizations, most importantly a bulk aerodynamic formulation for the surface fluxes and warm-rain microphysics. For a detailed description of the RAMS and the experimental setup the reader is referred to RMN. Our experiment considers a TC that has been spun up in quiescent environment on an f-plane for $48 \mathrm{~h}$. After this time the TC has reached an intensity ${ }^{5}$ of $68 \mathrm{~ms}^{-1}$ and is suddenly exposed to a zonal wind profile with vertical shear. The zonal wind profile has a cosine structure in the vertical with zero winds at the surface and $15 \mathrm{~ms}^{-1}$ easterlies at $12 \mathrm{~km}$ and above. After the environmental vertical shear flow is imposed, the TC starts moving to the west, with a small southward component, with an approximate translation speed of $5 \mathrm{~ms}^{-1}$.

Distinct changes in the TC structure occur a few hours after shear is imposed. The structural changes and their important connection to the thermodynamic impact on the TC inflow layer have been examined in detail in RMN. In response to the vertical shear forcing the TC vortex settles quickly into a quasi-equilibrium tilt direction approx. $90^{\circ}$ left of the shear vector $^{6}$. Such a tilt equilibrium may be achieved when the cyclonic precession of the tilted vortex cancels the differential advection by the vertical shear flow (Jones, 1995; Reasor et al., 2004). The vorticity anomaly associated with the tilt of the outer vortex at low levels contributes to the formation of a pronounced convective asymmetry outside of the eyewall, reminiscent of the stationary band complex (SBC) defined by WMF84. The SBC extends outwards up to a radius of approx. $180 \mathrm{~km}$ and wraps from the downshear-right at low levels to the downshear to downshear-left quadrants at upper levels. Strong and persistent, vortex-scale downdrafts form underneath the helical SBC updrafts. These downdrafts flush the inflow layer with low- $\theta_{e}$ air in the downshear-left semicircle. RMN argue that, to first order, it is this flushing of the inflow layer with low $\theta_{e}$ that governs the intensity evolution of the vertically sheared TC. While the TC in the reference run without vertical wind shear continues to intensify rapidly, the intensity of the TC in the $15 \mathrm{mps}$ case remains approximately constant in the first $8-10 \mathrm{~h}$ after the shear is imposed. With ongoing flushing of low- $\theta_{e}$ air into the inflow layer, TC intensity decreases by $10 \mathrm{~ms}^{-1}$ in the subsequent $6-8 \mathrm{~h}$. Associated with a gradual recovery of the inflow layer $\theta_{e}$, the TC then starts to re-intensify.

\subsection{Analyzed flow structure}

The correct frame of reference is of immanent importance for the analysis of the Lagrangian flow structure under the assumption of steady flow (see, e.g., Fig. 3 of Dunkerton et al., 2009 and the accompanying discussion on p. 5624-5625). In our case, this is the frame of reference moving with the

\footnotetext{
${ }^{5}$ In RMN and in this study, intensity is defined as the maximum azimuthal mean tangential wind at $1 \mathrm{~km}$ height.

${ }^{6}$ In RMN, the tilt is defined as the vector difference between the location of the vorticity centroids at $10 \mathrm{~km}$ and $1 \mathrm{~km}$ height.
} 
mature storm. We thus use data in a storm-relative frame of reference. A square domain of $2450 \mathrm{~km}$ side length centered on the $\mathrm{TC}^{7}$ is considered, using data from the innermost grid ( $5 \mathrm{~km}$ resolution) where available and from the outer domains ( $15 \mathrm{~km}$ and $45 \mathrm{~km}$ resolution, respectively) elsewhere. As discussed in the introduction, for the purposes of this investigation the flow outside the inner core in the numerical experiment can be meaningfully approximated as steady for $\mathcal{O}$ (1 day). This steady flow is represented here by a $6 \mathrm{~h}$ time average over the period from $3-8 \mathrm{~h}$ after the shear was imposed.

The algorithm of Ide et al. (2002) is applied to linearize the velocity field in the vicinity of the stagnation point and to determine the location of the stagnation point. The stable (unstable) manifolds are found by backward (forward) integration of the steady, layer-wise horizontal velocity field, using a second-order Runge-Kutta scheme with a spatial increment of $2.5 \mathrm{~km}$. The manifolds are seeded along the eigendirections of the linearized velocity field at a distance of 1 grid point $(5 \mathrm{~km})$ from the stagnation point.

\subsubsection{Overview}

We first provide an overview of the flow topology before examining its relation to the distribution of high- and low- $\theta_{e}$ air in the vicinity of the TC. The flow topology at low-levels above the inflow layer $(z=2 \mathrm{~km})$, at mid-levels $(z=5 \mathrm{~km})$, at upper-levels $(z=8 \mathrm{~km})$, and just below the outflow layer $(z=10 \mathrm{~km})$ will be considered in the following. The radial profiles of the azimuthally averaged tangential winds at these levels are shown in Fig. 2. As can be expected, the maximum tangential winds, as well as the tangential winds at outer radii, decrease with height. The shape of the radial profile is similar at all 4 levels.

The storm-relative environmental flow, calculated as the average storm-relative flow between radii of $200 \mathrm{~km}$ and $1000 \mathrm{~km}$, is $4.5 \mathrm{~ms}^{-1}$ from approx. west at $2 \mathrm{~km}, 1.5 \mathrm{~ms}^{-1}$ from approx. south at $5 \mathrm{~km}$, and $6.5 \mathrm{~ms}^{-1}$ and $8 \mathrm{~ms}^{-1}$ from approx. east at $8 \mathrm{~km}$ and $10 \mathrm{~km}$, respectively. The storm-relative environmental flow changes direction by approx. $180^{\circ}$ between low- and upper-levels and is minimized at mid-level which is characteristic of a vertical shear profile with a vertical wave number 1 structure. The strength of the tangential flow and the magnitude of the storm-relative flow at $2 \mathrm{~km}$ height in our numerical experiment is comparable to one of the cases considered by WMF84 (Hurricane David (1979), their Fig. 2), except for the direction of the storm-relative environmental flow. As mentioned by WMF84, the flow topology can be reoriented to adjust for this difference.

The flow topology in our experiment and its vertical structure are shown in Fig. 3. The flow topology in WMF84's

\footnotetext{
${ }^{7}$ The TC center is defined as the centroid of vorticity averaged over the lowest $2 \mathrm{~km}$ in a square with a side length of $120 \mathrm{~km}$ around the surface pressure minimum.
}

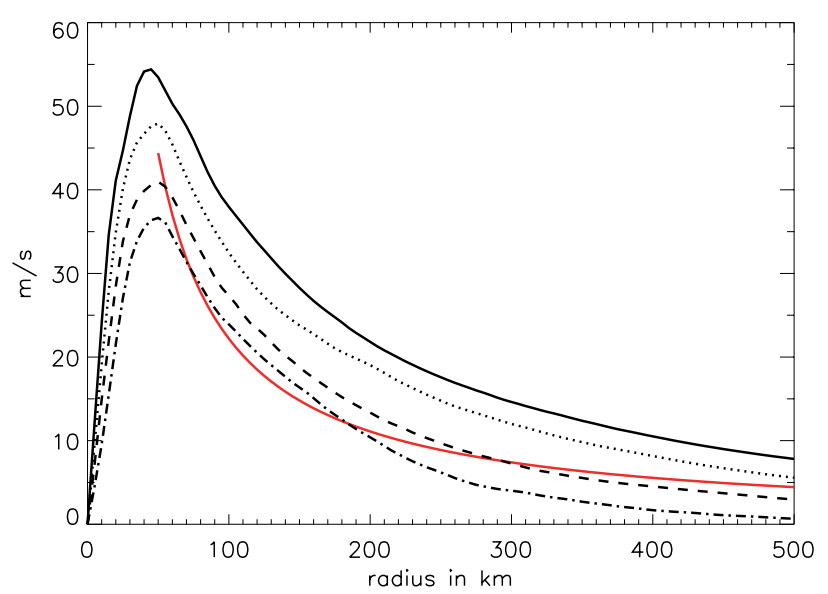

Fig. 2. Azimuthally averaged radial profiles of tangential winds for the no_shear case of RMN at $2 \mathrm{~km}$ (solid), $5 \mathrm{~km}$ (dotted), $8 \mathrm{~km}$ (dashed), and $10 \mathrm{~km}$ (dash-dotted), averaged over a $6 \mathrm{~h}$ period after $48 \mathrm{~h}$ of spin up. These profiles represent the TC wind structure at the time at which shear is imposed. For comparison, the profile for the Cat1 point vortex (red, see Sect. 3.2.1) is shown outside of $50 \mathrm{~km}$.

schematic (Fig. 1) was derived from winds at $850 \mathrm{hPa}$ and is thus comparable to our results at $2 \mathrm{~km}$ height (Fig. 3a). The signature and location of the SBC in the schematic of WMF84 was derived from radar reflectivities and satellite imagery and is thus more representative of vertical motion at mid- to upper levels (e.g. at $5 \mathrm{~km}$ and $8 \mathrm{~km}$ in our Fig. 3) than of the low-level vertical motion at $2 \mathrm{~km}$ height. After reorientation of WMF84's schematic by $180^{\circ}$ to adjust for the differences in the direction of the storm-relative environmental flow, a comparison with our results at $2 \mathrm{~km}$ height (Fig. 3a) reveals some notable differences. The stagnation point is found at a radius of approx. $700 \mathrm{~km}$ in our case, considerably farther outside than hypothesized by WMF84. One unstable manifold spirals inwards because the flow is weakly convergent at this level. This manifold ends in a so-called limit cycle, a closed streamline encompassing the strong updrafts in the inner core. In this case, the limit cycle is analogous to the closed streamline that encompasses the inner core and contains the "vortex air" in WMF84's hypothesized flow topology. In contrast, the outer portion of the manifolds are located at a considerably larger radius. These differences can be expected to be less pronounced for less intense TCs (see Eq. (13) below).

At $5 \mathrm{~km}$ (Fig. 3b) and $8 \mathrm{~km}$ (Fig. 3c) height the vortex is weakly convergent as can be inferred from the inward spiraling unstable manifold. At $10 \mathrm{~km}$ height (Fig. 3d), the flow is very weakly divergent indicating the transition to the well developed outflow layer above (not shown). As can be expected for the weaker storm-relative flow at $5 \mathrm{~km}$ height, the stagnation point is located at a larger radius (approx. $1000 \mathrm{~km}$ ) than 

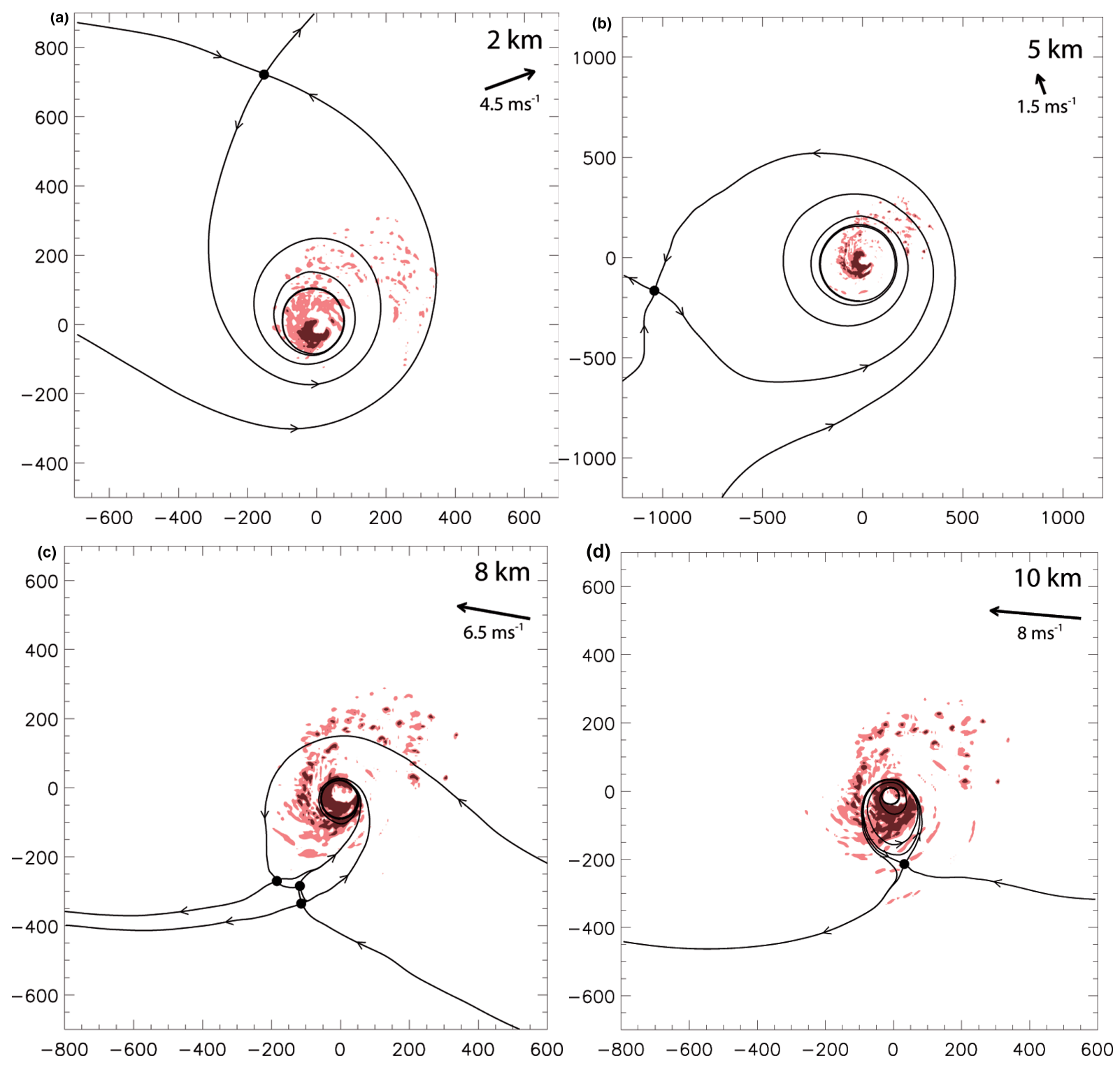

Fig. 3. Stagnation points (black dots) and manifolds at $2 \mathrm{~km}(\mathbf{a}), 5 \mathrm{~km}(\mathbf{b}), 8 \mathrm{~km}(\mathbf{c})$, and $10 \mathrm{~km}$ (d) height. The eyewall updrafts are indicated by the dark red shading $\left(1 \mathrm{~ms}^{-1}\right)$, light red shading denotes vertical motion of $0.25 \mathrm{~ms}^{-1}$. The magnitude and direction of the storm-relative flow is given in the upper-right of each panel. The values are rounded to $0.5 \mathrm{~ms}^{-1}$. The storm-relative environmental flow is calculated as the averaged flow between $200 \mathrm{~km}$ and $1000 \mathrm{~km}$ radius. The storm-relative asymmetric flow in this region is essentially uniform (not shown). All fields are from the $15 \mathrm{mps}$ run of RMN and averaged from $3-8 \mathrm{~h}$. The horizontal scale is in km; note the larger scale at $5 \mathrm{~km}$ height in (b).

at $2 \mathrm{~km}$ height. Consistent with storm-relative flow from the south, the stagnation point is located to the west. The inner core is encompassed by a large limit cycle of approx. $150 \mathrm{~km}$ radius. At $8 \mathrm{~km}$ and $10 \mathrm{~km}$ height, the stagnation points are located to the south and within $200 \mathrm{~km}-300 \mathrm{~km}$ from the center. Again, the location of the stagnation points is consistent with the direction and the stronger magnitude of the stormrelative environmental flow, and the weaker swirling winds at these levels as compared to $2 \mathrm{~km}$ and $5 \mathrm{~km}$ height. At $8 \mathrm{~km}$ height the limit cycle just encloses the eyewall updrafts. At
$10 \mathrm{~km}$ height, the manifolds form a virtually closed streamline that contains the eyewall.

It is interesting to note that there are multiple stagnation points at $8 \mathrm{~km}$. These multiple stagnation points are not an artefact of the time averaging of the velocity field but are found at individual times also (not shown). The occurrence of multiple stagnation points here does not imply a change of the system-scale flow topology. From each stagnation point one unstable manifold spirals inwards and merges into the limit cycle. 

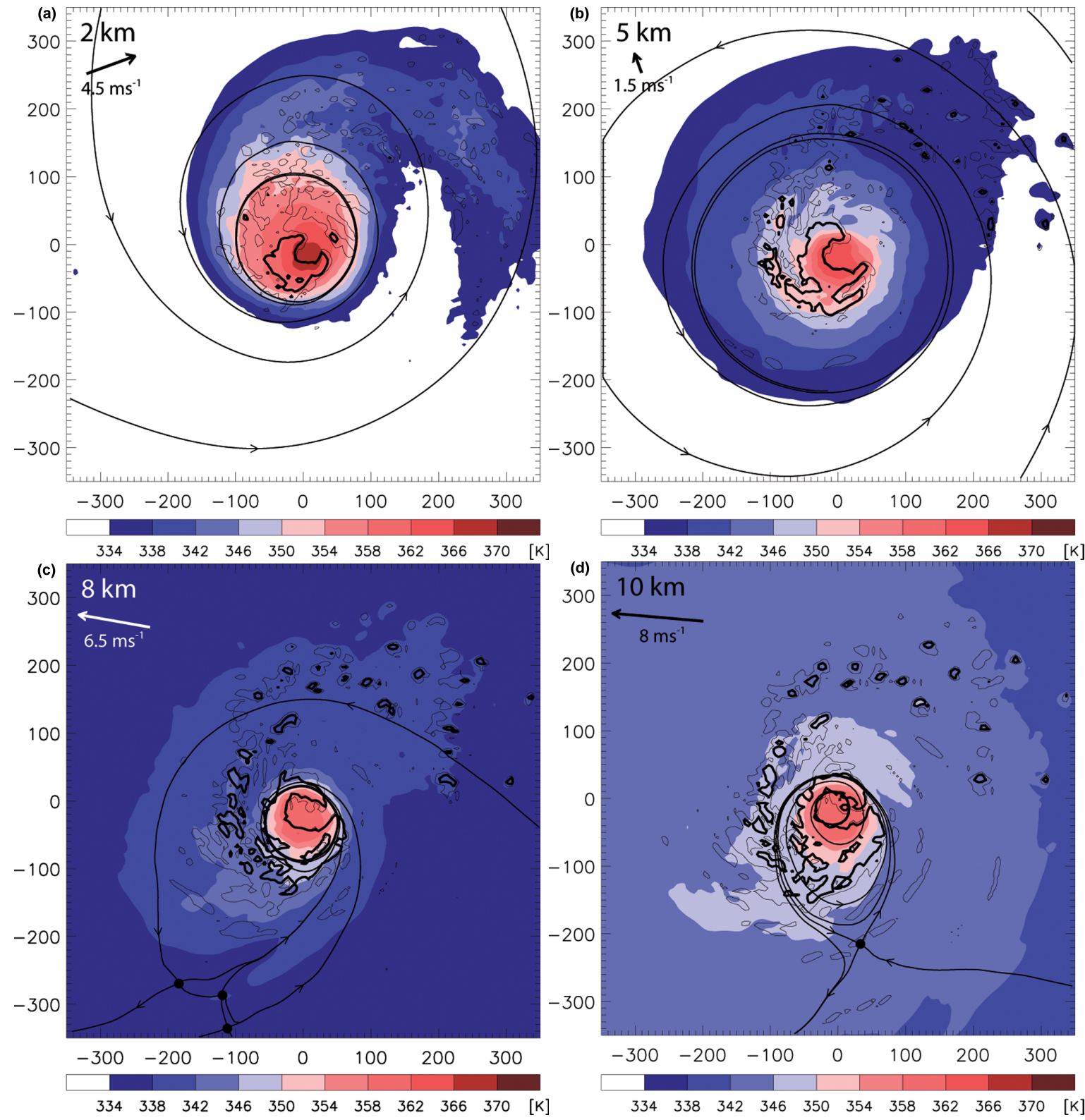

Fig. 4. Zoomed-in version of Fig. 3, including $\theta_{e}$ (color shaded) and vertical motion (thin contour $=0.25 \mathrm{~ms}^{-1}$, thick contour $=1 \mathrm{~ms}^{-1}$ ).

The analyzed flow topology is virtually identical when time averages from $2-7 \mathrm{~h}$ and $4-9 \mathrm{~h}$, respectively, are used. The general characteristics of the flow topology shown in Fig. 3 are also found for the $6 \mathrm{~h}$ periods from $10-15 \mathrm{~h}$ and $16-21 \mathrm{~h}$. The temporal differences in the details of the flow topology are discussed in Sect. 2.1.3.

\subsubsection{The meso-scale environment of the eyewall}

A zoomed-in version of Fig. 3, overlaid with $\theta_{e}$ (Bolton, 1980) at each respective height level, is shown in Fig. 4. A striking feature is that the distribution of $\theta_{e}$ is closely related to the manifold structure above the inflow layer up to $10 \mathrm{~km}$ height. Air with the highest values of $\theta_{e}$ are contained within the limit cycle ${ }^{8}$ ( $2 \mathrm{~km}-8 \mathrm{~km}$ height) or within the effectively closed streamline at $10 \mathrm{~km}$ height.

At $2 \mathrm{~km}$ height a clear wave number 1 asymmetry in the $\theta_{e}$ distribution is found (Fig. 4a). Very low values of $\theta_{e}$ are found within a radius of $100 \mathrm{~km}$ south of the center. To the north of the center, high values of $\theta_{e}$ are found within the

\footnotetext{
${ }^{8}$ The limit cycle is a robust feature of the flow topology derived from instantaneous snapshots of the storm-relative flow at individual times (hourly from $3 \mathrm{~h}$ to $8 \mathrm{~h}$ ).
} 
limit cycle and the innermost spiral, extending to a radius of $150 \mathrm{~km}$. In contrast, at $5 \mathrm{~km}$ height the $\theta_{e}$ distribution in the inner core is approximately symmetric, consistent with the shape and location of the limit cycle at this level (Fig. 4b). At $8 \mathrm{~km}$, the highest $\theta_{e}$ values outside of the eyewall are found southwest of the center, approximately bounded by the manifolds. At $10 \mathrm{~km}$, air with $\theta_{e}$ values of $342 \mathrm{~K}$ and lower appears to approach the TC from upshear. The eyewall and the highest $\theta_{e}$ values, however, are still encompassed by the closed streamline. The vertical motion field indicates that updrafts within the SBC provide higher $\theta_{e}$ air from below that is then advected to the downshear side with the quasi-steady flow.

Since $\theta_{e}$ tends to be a materially conserved quantity above the surface layer, $\theta_{e}$ is an approximate invariant of the timedependent, three-dimensional flow. Figure 4 shows a high degree of congruence between the $\theta_{e}$ isopleths and the manifolds of the steady, horizontal flow, in particular at low to mid-levels. Based on this notion we conclude that the shape and location of the moist envelope, i.e. the distribution of high- and low $-\theta_{e}$ air around the TC, is governed to first order by the stirring of convectively modified air by the steady, horizontal flow.

The existence of a closed streamline encompassing the eyewall clearly shows that the eyewall is well protected from the intrusion of environmental low- $\theta$ air by the steady, horizontal flow. In order for the environmental air to intrude into the inner core convection, time-dependent and/or vertical motions are needed. At upper-levels $(8 \mathrm{~km}$ and $10 \mathrm{~km})$, the manifolds barely encompasses the eyewall. This close approach of the manifolds to the eyewall indicates a potential "hot spot" for the interaction of the eyewall updrafts with environmental air. Note, however, that the highest $\theta_{e}$ values are well contained within the limit cycle. In particular, there is no indication that the eyewall "leaks", i.e. that high$\theta_{e}$ air is consistently transported downshear from out of the eyewall. Furthermore, from the perspective of the simplified Carnot-cycle model, the impact of ventilation on TC intensity is weighted by the difference between the outflow temperature and the temperature at the ventilation level. Ventilation at upper levels is thus not very effective in decreasing TC intensity (Tang and Emanuel, 2010b). In the following we therefore focus on the interaction of environmental air at low- to mid-levels.

\subsubsection{Time consistency}

The details of the flow topology exhibit some variability for the $6 \mathrm{~h}$ time-averaged periods from $10-15 \mathrm{~h}$ and $16-21 \mathrm{~h}$. For the sake of brevity we do not present the respective figures but provide a brief description of the salient features.

For the $6 \mathrm{~h}$ period from $10-15 \mathrm{~h}$ the unstable manifold spirals inwards very slowly at $2 \mathrm{~km}$ height and ends in a limit cycle that is larger than in Fig. 4a. This larger limit cycle encompasses all of the $\theta_{e}$ air with values larger than $342 \mathrm{~K}$.

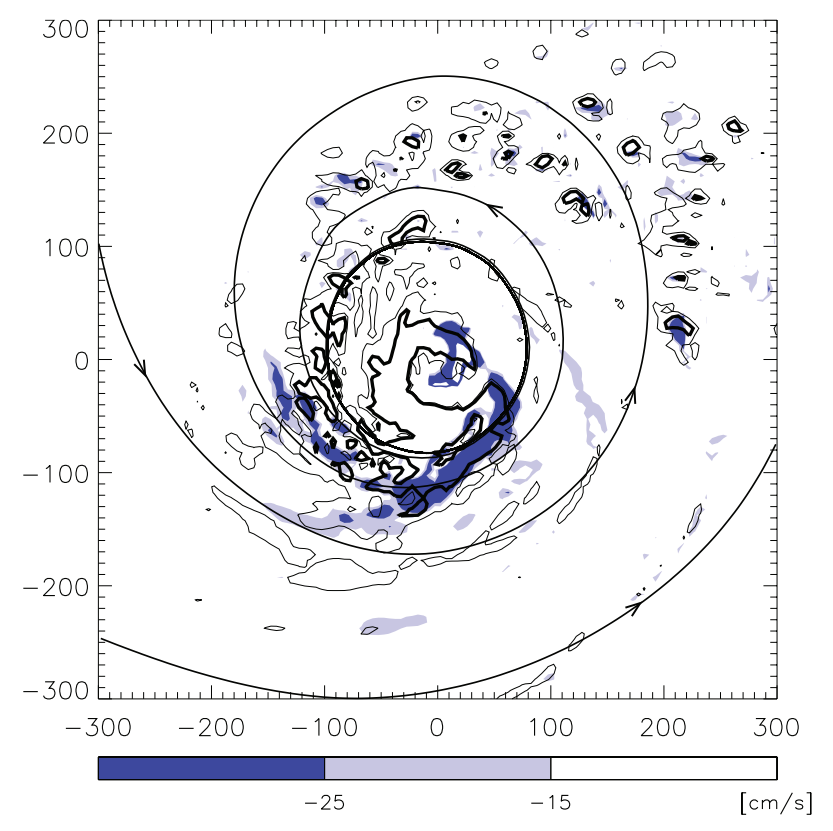

Fig. 5. Downdrafts (colored) and flow topology at $2 \mathrm{~km}$ and updrafts at $8 \mathrm{~km}$ (contours, thin $=0.25 \mathrm{~ms}^{-1}$, thick $=1 \mathrm{~ms}^{-1}$ ). Note that the limit cycle appears as a "thick contour" also because the manifold is plotted several times in a very similar location. The horizontal scale is in $\mathrm{km}$.

At $5 \mathrm{~km}$ height there is no stagnation point found within the domain under consideration. This is plausible because the steering level of the TC is expected to be around $5 \mathrm{~km}$ height. The storm-relative environmental flow is small at this height and consequently the stagnation point located at a very large radius (Eq. 13). At $8 \mathrm{~km}$ height, two stagnation points are found, located in the same region as shown in Fig. 3c. From each of these stagnation points one unstable manifold spirals around the storm center once before reaching the limit cycle. At $10 \mathrm{~km}$ height, the flow is weakly convergent at this time. Three stagnation points are analyzed from which 2 unstable manifolds spiral around the center. One manifold reaches the limit cycle encompassing the eyewall directly while the other spirals around the center once before merging into the limit cycle. The stable manifolds encompass the eyewall approx. $50 \mathrm{~km}$ further outside than during the period from 3-8h.

For the $6 \mathrm{~h}$ period from $16-21 \mathrm{~h}$ the flow topology at the individual levels is again very similar as during the period from 3-8 h, except at $5 \mathrm{~km}$ height. There, the unstable manifold spirals slowly inwards and merges into a limit cycle with a radius of approx. $60 \mathrm{~km}$.

Although there is some variability in the details of the flow topology at different times, the close relationship between the general manifold structure and the distribution of $\theta_{e}$ is verified for all time periods. Therefore, the assumption of 


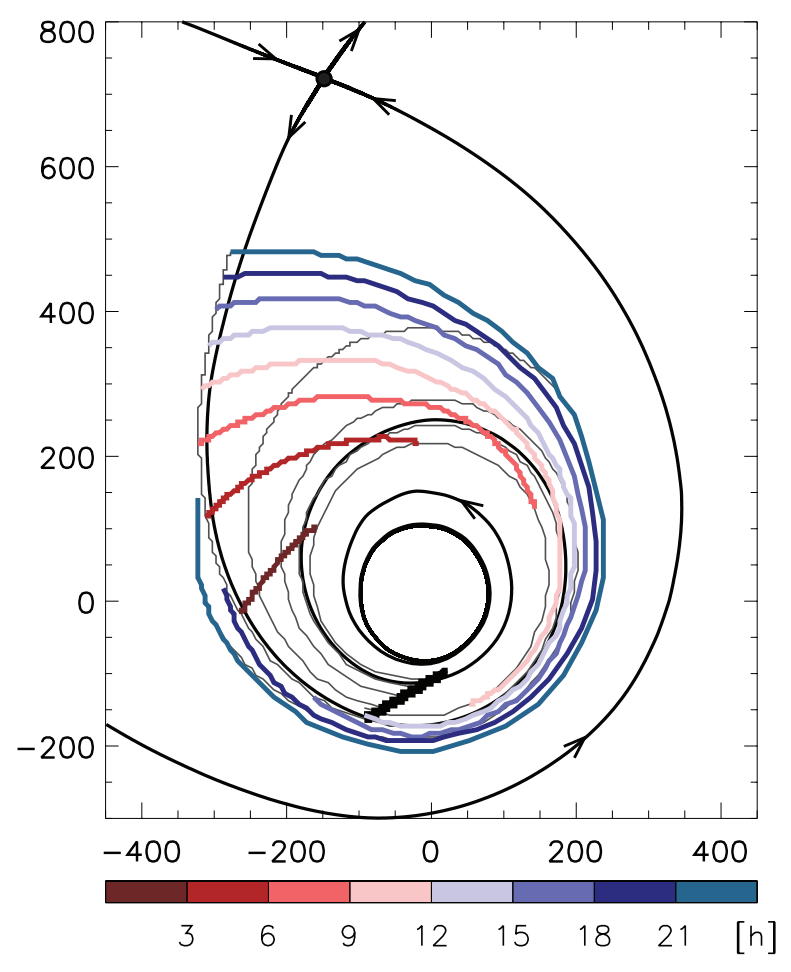

Fig. 6. Backward trajectories at $2 \mathrm{~km}$ seeded along a line segment (thick black line south of the center) approximating the central area of the downdraft region shown in Fig. 5. The colors denote the time it takes to reach the downdraft region. The thin lines exemplify some individual streamlines. The horizontal scale is in $\mathrm{km}$.

steady flow appears to be justified and the interpretation of the flow topology in terms of distinct streamlines is physically meaningful.

\subsection{Feeding of downdrafts by environmental air}

As discussed in RMN, persistent, vortex-scale downdrafts form in the downshear to downshear-left quadrant where precipitation from the helical SBC updrafts falls into the unsaturated air below. Further insight into the formation of these downdrafts can be obtained by examining the flow topology. Figure 5 indicates that the majority of the downdrafts occur outside of the limit cycle in a region of very low $\theta_{e}$ air at $2 \mathrm{~km}$ height (see Fig. 4a).

The manifold structure and $\theta_{e}$ distribution in Fig. 4a indicates that the downdraft region is continuously fed with very low $\theta_{e}$ air from the environment. This flow configuration appears to have two important ramifications. The continuous supply of dry, environmental air prevents the saturation of the air in the downdraft region due to the evaporation of precipitation. It is therefore plausible that the downdraft pattern may persist as a quasi-steady feature. Secondly, the flow pattern indicates that the environmental air reaches the downdraft re- gion with very little thermodynamic modification. Evaporation of precipitation from the SBC may thus occur in very dry air, leading to the formation of particularly vigorous downdrafts and an associated pronounced depression of the inflow layer $\theta_{e}$.

\subsection{Origin of environmental air fed into downdrafts}

The manifolds of the steady flow identify distinct flow regions and their interaction in the limit of long time scales. For the time scale relevant for TC-environment interaction of $\mathcal{O}$ (1 day) only subregions of the flow domain will be involved in such interaction. The extent of these subregions depend on the structure and magnitude of the velocity field. To estimate the origin of the air that is fed into the region of persistent downdrafts we calculate backward trajectories of the steady flow. Note that this steady flow here is represented by a $6 \mathrm{~h}$ time average. The backward trajectories are seeded at $2 \mathrm{~km}$ height along a line segment to the south of the storm center that approximates the central area of the downdraft region (Fig. 6). The color coding in Fig. 6 denotes where the air parcels are located at $3 \mathrm{~h}, 6 \mathrm{~h}, 9 \mathrm{~h}, \ldots$ before reaching the line segment.

Figure 6 illustrates that the source region of the environmental air that feeds the downdraft area exhibits a pronounced asymmetric, azimuthal wave number 1 structure. For the first $12 \mathrm{~h}$, the air originates almost exclusively from the west and north of the model TC. Subsequently, air progressively reaches the downdraft region that has spiraled around the center once. Ultimately, this air has its origin west of the TC also, as can readily be inferred from the manifold structure. For the first $24 \mathrm{~h}$, the source region of air interacting with the downdraft region from the south and east of the center is confined to a small radial area (approx. $150 \mathrm{~km}-$ $200 \mathrm{~km}$ ). This radial area is considerably smaller than indicated by the stable manifold, which is located at a radius of approx. $300 \mathrm{~km}$ south and east of the center. The potential ramification of this pronounced asymmetry of the source region of environmental air on TC intensity will be discussed in Sect. 4.3.

\section{A potential-flow model for TC-environment interaction}

Here we develop a simple analogue model that captures several principal features of the flow topology presented in the foregoing discussion based on the idealized experiment. Our analogue model is based on the well-known properties of a point vortex (e.g. Lamb, 1945) immersed in background flow, with a relatively weak mass sink. The model is in the same spirit of Smith et al. (2000) who also used a point vortex model to elucidate certain aspects of a TC in vertical shear. To our knowledge, the simple model has not been 
considered previously to examine the interaction of TCs with their environment.

\subsection{A convergent point vortex immersed in a uniform background flow}

We first consider a mass sink that is co-located with the point vortex.

\subsubsection{Streamfunction and wind components}

Consider first a point vortex at the origin $\mathbf{0}$ with circulation $\Gamma$ in a polar coordinate system with position vector $\boldsymbol{x}=(r, \phi)$, where $\phi$ increases in the anti-clockwise direction. The vertical vorticity is then given by

$\zeta(\boldsymbol{x})=\Gamma \delta(\boldsymbol{x})$,

where $\delta(\boldsymbol{x})$ is the two-dimensional Dirac delta function. The well-known associated streamfunction in the horizontal plane is

$\Psi_{v}(r, \phi)=\frac{\Gamma}{2 \pi} \ln r$,

and the tangential wind

$v_{v}=\frac{\partial \Psi_{v}}{\partial r}=\frac{\Gamma}{2 \pi r}$

with correspondingly zero radial wind.

Now, instead, consider a point mass sink of strength $D$ at the origin. This mass sink has divergence

$\mathcal{D}(\boldsymbol{x})=D \delta(\boldsymbol{x})$.

Because the flow associated with this mass sink is everywhere non-divergent save for this singularity, the following streamfunction can be defined ${ }^{9}$ :

$\Psi_{\text {div }}(r, \phi)=-\frac{D}{2 \pi} \phi$.

Because of our desire to readily visualize the flow, we prefer the use of a streamfunction over the velocity potential. The associated radial wind

$u_{\mathrm{div}}=-\frac{\partial \Psi_{\mathrm{div}}}{r \partial \phi}=\frac{D}{2 \pi r}$.

The tangential velocity is identically zero. Hereafter, we will absorb the $2 \pi$ into the definitions of $\Gamma$ and $D$, viz., $\Gamma^{*}=$ $\Gamma / 2 \pi$ and $D^{*}=D / 2 \pi$, and drop the * notation.

Since the problem of a point vortex in uniform background flow is invariant under (static) rotation we are free to choose the direction of $\mathrm{U}$. We choose the background flow to be zonal and define $\phi=0$ to be south. Let positive $U$ denote westerly flow. Cyclonic and outward flow with respect to the

\footnotetext{
${ }^{9}$ The streamfunction is a multi-valued function for which the Riemann sheets connect at $0 / 2 \pi$.
}

origin is defined to be positive. The streamfunction associated with the background flow is then

$\Psi_{\mathrm{bg}}(r, \phi)=U r \cos \phi$

and the tangential and radial wind components, respectively, are given by

$v_{\mathrm{bg}}=U \cos \phi$

and

$u_{\mathrm{bg}}=U \sin \phi$.

\subsubsection{Total streamfunction and flow visualization}

Because potential flow satisfies the linear Laplace equation $\nabla^{2} \Psi=0$ outside of flow singularities we may superpose the individual solutions. The total streamfunction for a divergent point vortex at the origin immersed in uniform background flow is then:

$\Psi(r, \phi)=\Psi_{v}+\Psi_{\mathrm{div}}+\Psi_{\mathrm{bg}}=\Gamma \ln r-D \phi+U r \cos \phi$.

It is clear from this equation that the term associated with the mass sink $\left(\Psi_{\text {div }}\right)$ introduces a jump discontinuity ${ }^{10}$ of magnitude $2 \pi D$ when a streamline crosses the southern semi-axis at $0 / 2 \pi$. The streamfunction is therefore multi-valued. Still, Eq. (10) is meaningful to represent the flow field because it is differentiable and thus the wind field is unique. The hyperbolic manifolds and the streamlines of the flow can be readily visualized when one contour line is chosen to pass through the stagnation point and a contour interval of $2 \pi D$, or an integer fraction thereof, is used (Fig. 7).

\section{2 "TC-like" vortex strength and mass sink}

In this subsection we use observations and our idealized numerical experiments to give a range of values for $\Gamma$ and $D$ that best represent a TC in the point vortex framework.

\subsubsection{Tangential flow}

The tangential wind of the point vortex decreases as $r^{-1}$. It is well known that the radial decrease of tangential winds in mature TCs, $v_{\mathrm{TC}}$, outside of the radius of maximum winds (RMW) is generally less than $r^{-1}$. A reasonable estimate of this radial structure is

$v_{\mathrm{TC}}=I_{0} r^{-\gamma}$,

with $\gamma$ ranging between 0.4 and 0.7 (Mallen et al., 2005, and references therein), and $I_{0}$ is a constant denoting the storm

\footnotetext{
${ }^{10}$ The structure of the equation for the velocity potential $\Phi$ of the combined flow is essentially the same and thus a likewise discontinuity occurs if $\Phi$ were used instead of $\Psi$ to represent the mass source.
} 


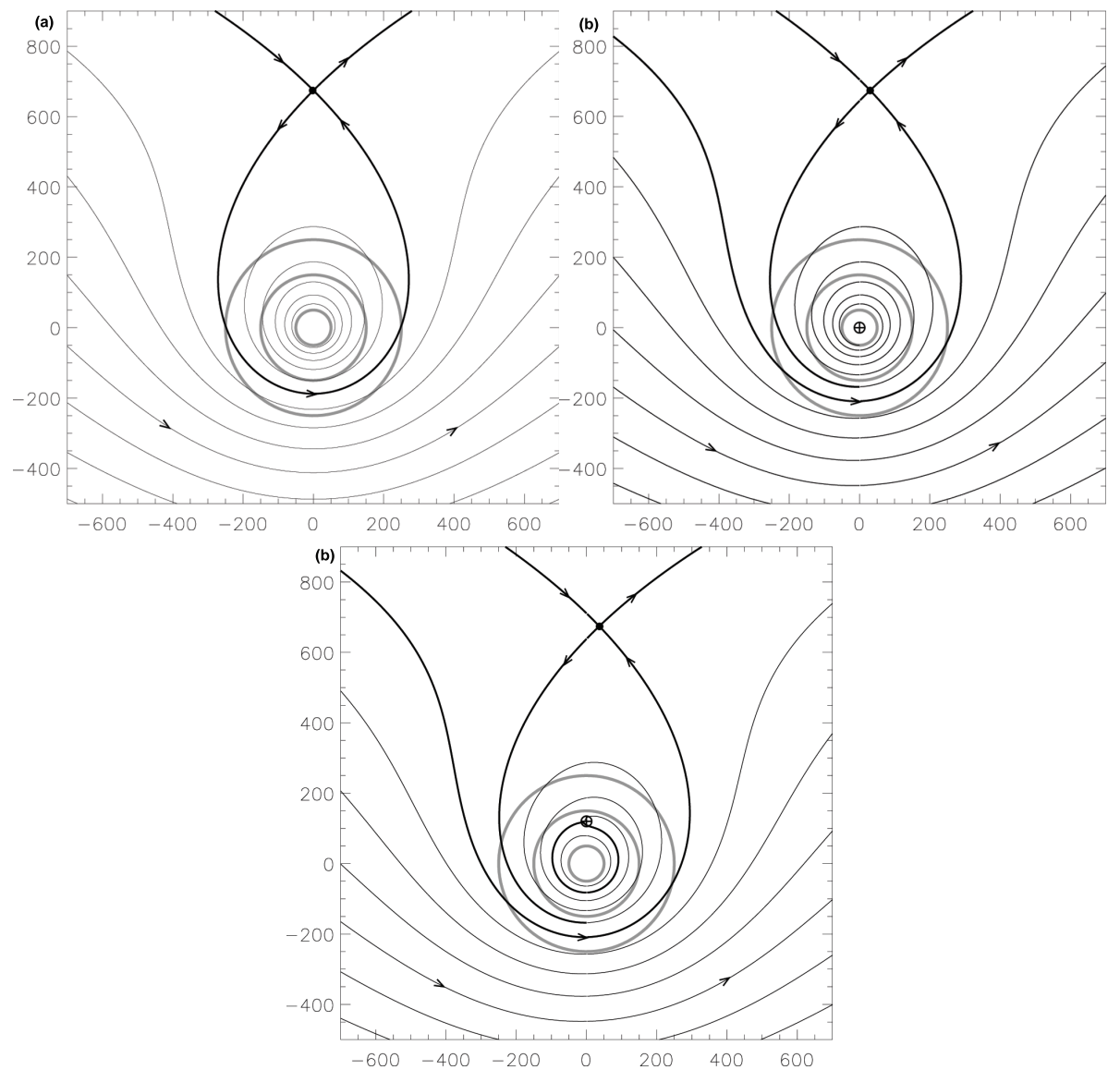

Fig. 7. (a) Streamlines of a stationary, cyclonic, non-divergent point vortex with a circulation of $33.7 \times 10^{5} \mathrm{~m}^{2} \mathrm{~s}^{-1}$ (Cat3, see Table 1 ) in $5 \mathrm{~ms}^{-1}$ westerly background flow. The strength of the vortex and the storm-relative environmental flow are chosen to be similar to the idealized numerical experiment at $2 \mathrm{~km}$ height (cf. Fig. 3). The streamlines passing through the stagnation point (black dot), the flow's manifolds, are highlighted. The horizontal scale is in $\mathrm{km}$. The gray concentric circles denote radii $50 \mathrm{~km}, 150 \mathrm{~km}$, and $250 \mathrm{~km}$, respectively. (b) Same as (a) but for a weakly convergent point vortex with $D=-1.5 \times 10^{5} \mathrm{~m}^{2} \mathrm{~s}^{-1}$. The mass sink is marked by the crossed circle. (c) Same as (b), but for a mass sink that is displaced $120 \mathrm{~km}$ to the north of the center. The emerging closed streamline, reminiscent of the limit cycle found in the idealized numerical experiment (cf. Fig. 3), is highlighted.

intensity. Much of the variability of $\gamma$ is believed to be associated with the stage of the TC life cycle ${ }^{11}$. In general, the radial decay of $v_{\mathrm{TC}}$ is slowest for storms at minimal hurricane strength or weaker. The radial decrease of tangential winds is somewhat steeper at upper-levels (Mallen et al., 2005). Due to the different radial profiles it can be expected that the details of the flow topology of a point vortex and a TC in background flow vary accordingly.

To investigate the general flow topology of the simple point vortex model in the context of TC-environment interaction, we believe that it is a reasonable choice to define the strength of the point vortex by its tangential winds at $150 \mathrm{~km}$. As is shown below (Sect. 3.3.1) this radius lies in between

\footnotetext{
${ }^{11}$ There is some variability of $\gamma$ with radius also, consistent with the well-known fact that the inner and outer wind fields can develop rather independently (Weatherford and Gray, 1988).
}

the radius of the stagnation point and the closest approach of the dividing streamline to the vortex center, except for strong vortices. Therefore, this choice appears to be a good compromise between underestimating the winds at larger radii and overestimating them at small radii. Accordingly, the associated circulation of the point vortex will be denoted by $\Gamma_{150}$.

The circulation of several vortices is defined to represent different TC intensities by the following procedure. We specify the maximum wind speed according to the National Hurricane Center (NHC) for a tropical depression ${ }^{12}$, tropical storm, and category 1 , category 3 , and category 5 hurricanes (Table 1). The corresponding point vortices are referred to as TD, TS, Cat1, Cat3, and Cat5, respectively. The RMW

\footnotetext{
${ }^{12}$ There is no official minimum wind speed requirement for the NHC to declare a tropical depression. Here we use a wind speed of $10 \mathrm{~ms}^{-1}$ to characterize a relatively weak closed circulation.
} 
Table 1. Definition of model vortices based on NHC TC intensity.

\begin{tabular}{lrrrrr}
\hline & TD & TS & Cat1 & Cat3 & Cat5 \\
\hline$V_{\mathrm{NHC}}\left[\mathrm{ms}^{-1}\right]$ & 10 & 17 & 33 & 50 & 70 \\
$\Gamma_{50}\left[10^{5} \mathrm{~m}^{2} \mathrm{~s}^{-1}\right]$ & 4.1 & 7.0 & 13.6 & 20.5 & 28.8 \\
$\Gamma_{150}\left[10^{5} \mathrm{~m}^{2} \mathrm{~s}^{-1}\right]$ & 6.7 & 11.5 & 22.2 & 33.7 & 47.2 \\
\hline
\end{tabular}

is set to $35 \mathrm{~km}^{13}$. We use Eq. (11) with $\gamma=0.55$ to calculate the tangential wind speed at $150 \mathrm{~km}$. This wind speed is then used in Eq. (3) to calculate the circulation $\Gamma_{150}$ for the individual vortex. Compared to the generic TC profile with $\gamma=0.55$, the point vortex overestimates the winds at $50 \mathrm{~km}$ by $64 \%$ and underestimates the winds at $500 \mathrm{~km}$ by $42 \%$. A comparison of the point vortex profile with the radial profile of the tangential winds in our numerical experiment is provided in Fig. 2.

To estimate whether environmental air may reach the eyewall, the overestimation of the tangential wind speed at the radius representing the eyewall is clearly problematic. For this estimate we consider the representation of the different TC categories by a modified circulation, $\Gamma_{50}$, also. To calculate $\Gamma_{50}$, the above procedure is repeated with the winds of the point vortex matched to the TC profile at a radius of $50 \mathrm{~km}$, instead of $150 \mathrm{~km}$. For the $\Gamma_{50}$ vortices, the approach of environmental air is likely overestimated because of the steeper radial wind profile of the point vortex as compared to the TC profile. Below, $\Gamma$ refers to $\Gamma_{150}$ unless otherwise noted.

The circulation of the point vortex may represent a TC of specified intensity up to mid-levels, around $5 \mathrm{~km}$. At upper-levels the tangential winds are considerably weaker. In conjunction with the faster radial decay of the wind speed (see above) the closest approach of environmental air to the core region can be expected at upper-levels below the outflow layer, consistent with the numerical results presented in Sect. 2.

\subsubsection{Radial flow}

The radial profile of the radial wind above the inflow layer is more variable and less documented observationally than the radial profile of the tangential winds. In this study, we use data from our previously published idealized numerical experiments (i.e., the no_shear, $15 \mathrm{mps}$, and $20 \mathrm{mps}$ case of RMN) to estimate values for $D$. We consider the temporal average from $4-9 \mathrm{~h}$. For all three experiments the radial flow at $150 \mathrm{~km}$ is approx. $1 \mathrm{~ms}^{-1}$ which, according to Eq. (6), yields a value of $D=-1.5 \times 10^{5} \mathrm{~m}^{2} \mathrm{~s}^{-1}$ for the point vortex model. At $50 \mathrm{~km}$, this value of $D$ yields a radial inflow velocity of $3 \mathrm{~ms}^{-1}$.

\footnotetext{
${ }^{13}$ This value underestimates the RMW for tropical depressions and potentially for tropical storms.
}

It needs to be pointed out, however, that the radial profiles of the TC in the idealized numerical experiments are not consistent with a point mass sink. For all three experiments the radial inflow does not increase inside $150 \mathrm{~km}$. In the no_shear and $15 \mathrm{mps}$ case a radial outflow of $5 \mathrm{~ms}^{-1}$ is found at $50 \mathrm{~km}$. There is considerable uncertainty associated with our choice of $D$. Nevertheless, the results of our analysis will be shown below to be only weakly sensitive to the choice of $D$ over a range of reasonable values.

\subsection{Flow topology}

Without loss of generality, we consider a cyclonic vortex $(\Gamma>0)$ in westerly background flow $(U>0)$.

\subsubsection{Non-divergent point vortex}

By definition, the stagnation point $\left(r_{\mathrm{sp}}, \phi_{\mathrm{sp}}\right)$ is found where both the tangential and radial flow components vanish. The radial flow for the non-divergent vortex vanishes everywhere. The radial component of the westerly background flow vanishes for $\phi=0$ and $\phi=\pi$, i.e. due south and due north of the center (Eq. 9). For a cyclonic vortex in westerly background flow the stagnation point is located to the north ${ }^{14}$, i.e. $\phi_{\mathrm{sp}}=\pi$. The condition for the cancellation of the tangential flow is

$v_{v}\left(r_{\mathrm{sp}}, \phi_{\mathrm{sp}}\right)+v_{\mathrm{bg}}\left(r_{\mathrm{sp}}, \phi_{\mathrm{sp}}\right)=\frac{\Gamma}{r_{\mathrm{sp}}}+U \cos \phi_{\mathrm{sp}}=0$.

Using $\phi_{\mathrm{sp}}=\pi$ in Eq. (12) gives the radius of the stagnation point

$r_{\mathrm{sp}}=\frac{\Gamma}{U}$.

As to be expected, for a weaker vortex and stronger background flow the stagnation point is found closer to the center. For a "Cat1" vortex in $5 \mathrm{~ms}^{-1}$ background flow the stagnation point resides at $444 \mathrm{~km}$.

The value of the streamfunction at the stagnation point is given by

$\Psi\left(r_{\mathrm{sp}}, \phi_{\mathrm{sp}}\right)=\Gamma\left(\ln \frac{\Gamma}{U}-1\right)$.

This value of the streamfunction designates the dividing streamline, i.e. the streamline that passes through the stagnation point. Note that within the closed gyre of the dividing streamline all streamlines form closed, cyclonic gyres (Fig. 7a). The closest approach of the dividing streamline to the point vortex is located on the opposite side of the stagnation point, i.e. south of the vortex. The radius of this closest approach for the non-divergent vortex, $R_{\mathrm{nd}}$, is found by

\footnotetext{
${ }^{14}$ In the Southern Hemisphere, the stagnation point is to the south.
} 
equating the streamfunction south of the vortex $(\phi=0)$ to the value of the dividing streamline:

$U R_{\mathrm{nd}}+\Gamma \ln R_{\mathrm{nd}}=\Gamma\left(\ln \frac{\Gamma}{U}-1\right)$.

Equation (15) can be written as

$\alpha+\ln \alpha=-1$

with $\alpha=R_{\text {nd }} U / \Gamma$. A solution to Eq. (16) can be found numerically ${ }^{15}: \alpha \approx 0.278$. Hence we can write

$R_{\mathrm{nd}}=\alpha \frac{\Gamma}{U}$

or in terms of the radius of the stagnation point

$R_{\mathrm{nd}}=\alpha r_{\mathrm{sp}}$

\subsubsection{Point vortex with mass sink}

We now obtain an expression for the stagnation point $\left(r_{\mathrm{sp}}^{\mathrm{div}}, \phi_{\mathrm{sp}}^{\mathrm{div}}\right)$ in the case of the divergent point vortex. Since the divergent flow does not project on the tangential flow, Eq. (12) still gives the condition for the cancellation of the tangential wind component. However, the condition for the cancellation of the radial wind components now is

$\frac{D}{r_{\mathrm{sp}}^{\mathrm{div}}}+U \sin \phi_{\mathrm{sp}}^{\mathrm{div}}=0$.

Combining Eqs. (12) with (19) yields:

$\phi_{\mathrm{sp}}^{\mathrm{div}}=\arctan (D / \Gamma)$.

Because the arc-tangent function returns values between $\pm \pi / 2$, Eq. (20) is best regarded as the deviation from the stagnation point in the non-divergent case $(\arctan (D / \Gamma)=0$ for $D=0$ ). The equation for the azimuth of the stagnation point in our case is then

$\phi_{\mathrm{sp}}^{\mathrm{div}}=\pi+\Delta$,

with $\Delta=\arctan (D / \Gamma)$. The azimuthal shift relative to the non-divergent case is governed by the ratio $D / \Gamma$, which is usually small for TC-like vortices. Under the condition that $D / \Gamma \ll 1$ we may linearize Eq. (20) to yield

$\Delta \approx D / \Gamma$.

It is interesting to note that the direction of the azimuthal shift depends only on the sign of $D / \Gamma$. For a convergent, cyclonic vortex $(D<0, \Gamma>0)$, the stagnation point is shifted anticyclonically with respect to the non-divergent vortex. Usually, $|D / \Gamma| \approx 0.1-0.2$. Therefore, the azimuthal shift of

\footnotetext{
${ }^{15}$ We have used Newton's method with 0.2 as the initial guess and a convergence threshold of $10^{-4}$. A formal solution to Eq. (16) is $\alpha=\mathcal{W}\left(e^{-1}\right)$, with the Lambert $\mathrm{W}$-function $\mathcal{W}$. The value of $\mathcal{W}\left(e^{-1}\right)$, however, cannot be given analytically (Roy and Olver, 2010).
}

the stagnation point is on the order of $5^{\circ}-10^{\circ}$. In Fig. 7, $|D / \Gamma| \approx 0.05$ resulting in a visible but small azimuthal shift of the stagnation point.

Inserting Eq. (21) into Eq. (12) yields

$r_{\mathrm{sp}}^{\operatorname{div}}=\frac{\Gamma}{U \cos \Delta}$

which again can be linearized for small $\Delta$ :

$r_{\mathrm{sp}}^{\operatorname{div}}=\frac{\Gamma}{U\left(1-\Delta^{2}+\ldots\right)}$.

To first order, the radius of the stagnation point does not change when a relatively small mass sink is introduced. To second order $r_{\mathrm{sp}}^{\mathrm{div}}$ increases over its non-divergent counterpart. The value for the streamfunction at the stagnation point

$\Psi\left(r_{\mathrm{sp}}^{\mathrm{div}}, \phi_{\mathrm{sp}}^{\mathrm{div}}\right)=\Gamma\left(\ln \frac{\Gamma}{U \cos \Delta}-1\right)-D(\pi+\Delta)$.

The above results show that the location of the stagnation point is not sensitive to the introduction of a mass sink that is small compared to the vortex strength. Nevertheless, the flow topology changes fundamentally. Due to the mass sink the formerly closed dividing streamline opens up. A direct pathway emerges through which environmental air can spiral towards the mass sink at the vortex center (Fig. 7). We can estimate the width $d$ of the opening as follows. In the steady case, the inflow through this opening balances the mass sink. Due south of the center we can write

$\int_{R_{i}}^{R_{o}} v(r) d r+2 \pi D=0$,

where $R_{i}$ and $R_{O}$ denote the radii of the inner (unstable) and outer (stable) manifold ${ }^{16}$, respectively, and $v$ the tangential flow. We may assume that the radial interval is centered on $R_{\mathrm{nd}}$. The value of the integral is then $U d+\Gamma \ln \left(\frac{R_{\mathrm{nd}}+d / 2}{R_{\mathrm{nd}}-d / 2}\right)$. Expanding the logarithm in a Taylor series and truncating at third order, Eq. (26) reads

$\left(U+\frac{\Gamma}{R_{\mathrm{nd}}}\right) d+2 \pi D=0$,

Using Eq. (17) we find

$d=-\frac{\alpha}{1+\alpha} \frac{2 \pi D}{U}$.

For the standard value $D=-1.5 \times 10^{5} \mathrm{~m}^{2} \mathrm{~s}^{-1}$ and $U=$ $5 \mathrm{~ms}^{-1}$, we find that $d \approx 40 \mathrm{~km}$. This value compares well with the width of the opening illustrated in Fig. 7. A close agreement between the value derived from Eq. (28) and the actual width has been verified for reasonable values of $U$ and $D$ (not shown). It is interesting to note that within this good

\footnotetext{
${ }^{16}$ For positive values of $D$, i.e. net divergence, the stable manifold spirals inwards and is located radially inside of the unstable manifold.
} 
approximation (Eq. 28) the opening between the manifolds depends on $U$ and $D$ only. It does not depend on the circulation $\Gamma$ of the vortex.

In comparison to the dividing streamline, the presence of the mass sink causes the inward spiraling manifold to move closer to the center. An estimate for this inward displacement is $d / 2$. The closest approach of this inner manifold in the divergent case, $R_{\text {div }}$, is then

$R_{\mathrm{div}}=R_{\mathrm{nd}}-d / 2$.

Accordingly, an estimate for the closest approach of the outer manifold

$R_{\mathrm{div}}^{\mathrm{out}}=R_{\mathrm{nd}}+d / 2$.

\subsection{Point vortex with "asymmetric" mass sink}

We do not attempt to give the analytic solution for a point vortex with a mass sink displaced from the center. We note that this flow configuration is not a steady-state solution because the mass sink would tend to be advected by the swirling winds of the point vortex. We use this flow configuration as an ad-hoc explanation for the occurrence of the limit cycle found in the flow topology of the idealized model TC (Fig. 3a).

The asymmetric convergence at low-levels in our idealized numerical experiment is associated with the SBC (not shown). At $2 \mathrm{~km}$ height, the SBC is located approximately north of the center. The centroid of convergence upon averaging azimuthally over the northern semicircle is at $120 \mathrm{~km}$. In the point vortex model the mass sink is therefore placed due north at $120 \mathrm{~km}$ radius.

For the special case of a mass sink located on the line segment between the origin and the stagnation point, the impact on the location of the stagnation point can be analyzed analytically. Let us denote the radial location of the mass sink as $R_{\text {asy }}$. By symmetry, the projection of the divergent flow on the tangential wind component vanishes at the stagnation point in this case and the condition for the cancellation of the tangential wind, Eq. (12), remains unaltered. Let $\left(r_{\mathrm{sp}}^{\mathrm{asy}}, \phi_{\mathrm{sp}}^{\mathrm{asy}}\right)$ denote the location of the stagnation point. The condition for the cancellation of the radial flow components (Eq. 19) then reads

$\frac{D}{\left(r_{\mathrm{sp}}^{\mathrm{asy}}-R_{\mathrm{asy}}\right)}+U \sin \left(\phi_{\mathrm{sp}}^{\text {asy }}\right)=0$,

which can be re-written as

$$
\frac{D_{\text {asy }}}{r_{\mathrm{sp}}^{\text {asy }}}+U \sin \left(\phi_{\mathrm{sp}}^{\text {asy }}\right)=0
$$

where

$$
D_{\mathrm{asy}}=D \frac{r_{\mathrm{sp}}^{\mathrm{asy}}}{r_{\mathrm{sp}}^{\mathrm{asy}}-R_{\mathrm{asy}}} .
$$

From Eq. (22) we see that

$$
\frac{\Delta_{\mathrm{sp}}}{\Delta_{\mathrm{sp}}^{\text {asy }}}=\frac{r_{\mathrm{sp}}^{\text {asy }}-R_{\mathrm{asy}}}{r_{\mathrm{sp}}^{\text {asy }}}=1-\frac{R_{\mathrm{asy}}}{r_{\mathrm{sp}}^{\text {asy }}} .
$$

The azimuthal shift of the stagnation point in the presence of an asymmetric mass sink is denoted by $\Delta_{\mathrm{sp}}^{\text {asy }}$. For a radial displacement of the mass sink that is small compared to the radius of the stagnation point, the change in azimuth of the stagnation point is small. As we have seen before, the radius of the stagnation point does not change to first order. Accordingly, the general structure of the flow topology outside of the radius of the mass sink is not sensitive to its precise location or strength.

A numerical solution for the streamfunction is obtained by shifting the divergent streamfunction (Eq. 5) to the location of the mass sink and then adding to the non-divergent solution. The same algorithm (Ide et al., 2002) as used for the numerical experiment in Sect. 2 is applied to find the precise location of the stagnation point. From this solution, the value of the streamfunction at the stagnation point is then determined.

Figure $7 \mathrm{c}$ verifies that the location of the stagnation point and the manifold structure outside of the radius of the mass sink is not sensitive to the displacement of the mass sink in the regime of TC-like point vortices (cf. Fig. 7). A closed streamline, however, now emerges radially just inwards of the asymmetric mass sink similar to the closed cyclonic gyres found in the non-divergent point vortex model (Fig. 7a). The flow within this closed streamline is effectively nondivergent because the streamline does not encompass the offcenter mass sink. The closed streamline is reminiscent of the limit cycle in the idealized experiment (cf. Fig. 3a). In the divergent point vortex model with divergence off-center, however, the unstable manifold spirals into the mass sink and does not end in the putative "limit cycle".

\section{Application of the point vortex model to TC-environment interaction}

The examination of the flow topology in the previous sections demonstrates that the potential of the storm-relative environmental flow to bring environmental air close to the TC center is considerably limited by the deflection due to the swirling winds. We now use the simple point vortex model to estimate for which combinations of TC intensity and stormrelative environmental flow pronounced environmental interaction may be expected.

To apply the point vortex model, we have to assume a generic TC structure and associated $\theta_{e}$ distribution above the boundary layer in quiescent environment. Particularly high values of $\theta_{e}$ are contained within the eye and the surrounding eyewall. A typical radial scale of the eyewall is $50 \mathrm{~km}$. The region adjacent to the eyewall is often dominated by pronounced rain band activity. A typical radial scale for 
this region might be related to the stagnation radius of radially propagating VRWs and is $\mathcal{O}(150 \mathrm{~km})$ (Chen and Yau, 2001). Rain bands mix high- $\theta_{e}$ air from the boundary layer into the mid- to upper troposphere, considerably increasing $\theta_{e}$ values in the free troposphere as compared to the synoptic scale environment. Beyond this region, there is likely a transition region where $\theta_{e}$ values are still higher than in the synoptic-scale environment. In this region, surface fluxes are enhanced due to the TC winds, which could trigger sporadic deep convection. Strong rain bands may extend into this region also, contributing to further moistening. Beyond this region, outside a radius of $\mathcal{O}(250 \mathrm{~km})$, we assume that the thermodynamic properties of the atmosphere above the inflow layer are no longer modified by the TC. Air originating in this region is hereafter referred to as "environmental air". The $\theta_{e}$ values of the environmental air may be representative of a "typical" tropical environment, or may exhibit particularly low $\theta_{e}$ values due to synoptic-scale subsidence, intrusion of midlatitude air, or the presence of SAL. We will consider the two regimes of environmental interaction discussed in the introduction: The direct interaction of environmental air with the eyewall and the interaction with rain bands.

The assumed $\theta_{e}$ distribution is based on the common notion that a TC in quiescent environment is embedded in a region of moist air, the "moist envelope" (Kimball (2006); S. A. Braun, personal communication, 2010) ${ }^{17}$. Consistent with these studies, we have chosen a typical radius for environmental air of $\mathcal{O}(250 \mathrm{~km})$. Our idealized numerical experiment generally supports the existence of a moist envelope, albeit of smaller radial extent (Fig. 4b). A radius of $250 \mathrm{~km}$ might therefore overestimate the extent of moist air around the TC. The tangential winds of the point vortex, on the other hand, decay more rapidly with radius than for a typical TC wind profile (Sect. 3.2.1). Thus, environmental air in the point vortex model may penetrate closer to the center than for a more realistic radial wind profile. This sharper radial decrease of the tangential winds may therefore compensate to some degree for the potential overestimation of the radial scale of the moist envelope.

\subsection{A simple estimate of environmental interaction based on the location of the dividing streamline}

A simple estimate of potential environmental interaction can be derived from the location of the dividing streamline. For now, we assume that for a vertically sheared TC the environmental air is located outside of the dividing streamline, as in WMF84. If the closest approach of the dividing streamline to the center is within the rain band $(150 \mathrm{~km})$ or eyewall $(50 \mathrm{~km})$ radius, then pronounced interaction with rain bands and eye-

\footnotetext{
${ }^{17}$ We have been unable to trace the origin of the term "moist envelope". The notion of a moist, meso-scale eyewall environment may originate from WMF84's result that the "vortex air" is confined to the vicinity of the TC by the swirling winds.
}

wall convection, respectively, can be expected. The closest approach of the dividing streamline is given by Eq. (17).

We first note that Eq. (17) provides theoretical support for the hypothesis in RMN that the "character" of shear-TC interaction scales with the ratio of TC intensity and shear magnitude. Here, the shear magnitude is represented by the storm-relative environmental flow $U$, as discussed in the introduction.

The storm-relative environmental flow that is necessary to move the dividing streamline to a specified radius $R_{\mathrm{nd}}$ increases linearly with $\Gamma$. For the range of values of $\Gamma$ representing TC intensities from TD to Cat5, we find distinct scenarios of potential environmental interaction (Fig. 8). Direct eyewall interaction seems likely for the TD and TS vortices for storm-relative flow starting at approx. $3 \mathrm{~ms}^{-1}$, while storm-relative environmental flow well above $10 \mathrm{~ms}^{-1}$ is necessary to move the dividing streamline to a radius of $50 \mathrm{~km}$ for the Cat 3 and Cat5 vortices. Estimating that the low-level storm-relative environmental flow is approximately half of the wind difference between $850 \mathrm{hPa}$ and $200 \mathrm{hPa}$, i.e. the deep-layer vertical "shear", it can be concluded that direct interaction of environmental air with the eyewall of TCs of tropical storm intensity and below is likely in weak to moderate deep-layer "shear" of $6 \mathrm{~ms}^{-1}$ and above. For mature TCs of category 3 or stronger, a direct interaction of environmental air with the eyewall is unlikely even in strong deep-layer "shear" of $20 \mathrm{~ms}^{-1}$. Note that we have used $\Gamma_{50}$ to derive this result. Due to the slower radial decay of the tangential winds in real TCs, these estimates are a lower bound of the required storm-relative flow. Using larger circulation values to represent the respective TC category, e.g. $\Gamma_{150}$, the differences between the weak and the strong TC categories are even more pronounced (not shown). For mature storms (category 3 and above), moderate to strong deep-layer shear is likely to promote pronounced interaction of environmental air with rain bands. Such interaction can produce persistent, vortex-scale downdrafts leading to a considerable weakening of the storm (Fig. 5 and RMN).

The results presented above suggest that it is not only the dynamic resiliency of a TC that increases with intensity (Jones, 1995; Reasor et al., 2004). The ability of a TC to isolate itself from adverse thermodynamic interaction with environmental air increases with intensity also. A similar point can be made for vortex size. A broad TC with a relatively large radius of gale force winds has evidently a larger circulation than a TC with the same maximum intensity but a smaller radius of gale force winds. Thus, it can be expected that the ability of a TC to thermodynamically isolate itself from the environment increases with TC size. Very distinct scenarios of possible environmental interaction in vertical shear are indicated for values of $U$ and $\Gamma$ that represent realistic TC conditions in this simplified framework. 


\subsection{An improved estimate of environmental interaction: inward flow rate of environmental air}

It is important to note that environmental air can reside within the dividing streamline also (e.g. Fig. 4a). Potential interaction with this environmental air is not accounted for by the estimate presented in the previous subsection. In our simple point vortex framework, environmental air resides within the dividing streamline when the stagnation radius is beyond $250 \mathrm{~km}$. For the Cat1 vortex, $r_{\mathrm{sp}}>250 \mathrm{~km}$ for $U<8.9 \mathrm{~ms}^{-1}$ (Eq. 13). Furthermore, the effect of weak convergence above the inflow layer has not been taken into account in Sect. 4.1.

We propose that the rate $\mathcal{F}_{\text {env }}$ by which environmental air is transported into the rain band or eyewall region provides an improved estimate for potential environmental interaction in our simple model. The volumetric flow rate $\mathcal{F}$ through an area bounded by a curve $\mathcal{L}$ and unit height

$\mathcal{F}=\int_{\mathcal{L}} v_{n} d l$,

where $d l$ is a line segment of $\mathcal{L}$ and $v_{n}$ is the flow normal to $d l$. The inflow rate of environmental air, $\mathcal{F}_{\text {env }}$, is determined from the streamlines that connect the environmental radius and the rain band and eyewall radius, respectively. Streamlines are calculated that emanate along the environmental radius $R_{\mathrm{env}}=250 \mathrm{~km}$ with a constant azimuthal spacing $\Delta \phi=3^{\circ}$. We select a subset $\mathcal{S}_{c}$ of these streamlines that exhibit radial inflow at $R_{\text {env }}$ and connect to the rain band (eyewall) radius without orbiting the center. The radial flow at radius $r$ associated with the $i$ th streamline of this subset is denoted by $u_{\mathcal{S}_{c}^{i}}(r)$. Due to mass conservation and the steadiness of the flow, $\mathcal{F}_{\text {env }}$ at the rain band (eyewall) radius equals the radial inflow rate at the environmental radius associated with $\mathcal{S}_{c}$ :

$\mathcal{F}_{\text {env }}=\sum_{i} u_{\mathcal{S}_{c}^{i}}\left(R_{\text {env }}\right) R_{\text {env }} \Delta \phi$

We exclude orbiting streamlines from our calculation because air parcels along such paths move inwards very slowly. It seems unreasonable to assume that the flow is approximately steady on this longer time scale. Air parcels that spiral through the rain band region towards the eyewall are likely to interact considerably with rain bands before reaching the eyewall. It can be assumed that this interaction moistens the environmental air considerably before eyewall interaction takes place. It is, however, an open question if convection outside of the eyewall is indeed vigorous enough to protect the inner core from such slow approach of environmental air.

The values of $\mathcal{F}_{\text {env }}$, calculated from the point vortex with centered mass sink, are depicted in Fig. 9 for a large part of the phase space relevant for TCs. For comparison, the values of $U$ and $\Gamma$ for which the closest approach of the dividing streamlines is at the rain band and eyewall radius, respectively, are indicated also. The dividing streamline apparently gives a good estimate for the onset of potential environmental interaction. In general, low values of $\mathcal{F}_{\text {env }}$ are found for values of $\Gamma$ and $U$ for which the dividing streamline is outside of the rainband (Fig. 9a) and eyewall radius (Fig. 9b), respectively. It is clear that $\mathcal{F}_{\text {env }}$ increases with increasing $U$ for a given circulation $\Gamma$. For eyewall interaction, the location of the dividing streamline denotes the onset of the inflow of environmental air extremely well (Fig. 9b). For the rain band region (Fig. 9a), potential environmental interaction is slightly underestimated for strong vortices. With this small caveat in mind, the phase space diagram of the dividing streamline location (Fig. 8) may be used as a first, simple guidance to estimate the propensity of TC-environment interaction.

\subsection{A well defined source region of environmental air}

As noted in Sect. 2.3 in the discussion of Fig. 6, the source region of environmental air interacting with a TC may exhibit a pronounced asymmetric, azimuthal wave number 1 structure. For a Cat 1 vortex with $U=5.1 \mathrm{~ms}^{-1}$ the envelope of streamlines that connect the environment to the rain band radius are overlaid on the flow topology in Fig. 10. It is evident that the main source region of the environmental air that is transported into the rain band region is to the northwest of the storm. Westerly storm-relative flow at low levels indicates that such a TC would be in easterly vertical wind shear.

Now consider a region of very dry air to the southeast of the storm (shaded region in Fig. 10). Although some of this air is located within $200 \mathrm{~km}$ of the TC center, the interaction of the dry air with the TC is greatly limited in the easterly wind shear scenario depicted in Fig. 10: the dry air is located outside of the stable manifold. If the vertical wind shear were westerly, however, the low-level storm-relative flow would be from the east. The pattern of the flow topology would be rotated by $180^{\circ}$ and would indicate a pronounced intrusion of the very unfavorable dry air masses into the TC circulation.

This brief discussion demonstrates that in the presence of moisture gradients the adverse impact of vertical wind shear on a TC may be sensitive to the direction of the shear. If the flow topology favors the interaction with particularly dry air masses, a more pronounced weakening of the TC can be expected as in comparison to a shear direction that favors interaction with relatively moist environmental air.

The interaction of TCs with the meridional gradient of planetary vorticity leads to the formation of broad-scale vorticity asymmetries, the so-called $\beta$-gyres. In a quiescent environment, the $\beta$-gyres lead to a southeasterly flow across the vortex center at low levels. This storm-relative flow in turn implies a northeasterly vertical wind shear on the TC (e.g. Bender, 1997; Ritchie and Frank, 2007). Ritchie and Frank have hypothesized that this so-called $\beta$-shear adds linearly to the environmental vertical shear over the storm center. Thus, these authors have proposed that TCs should be 


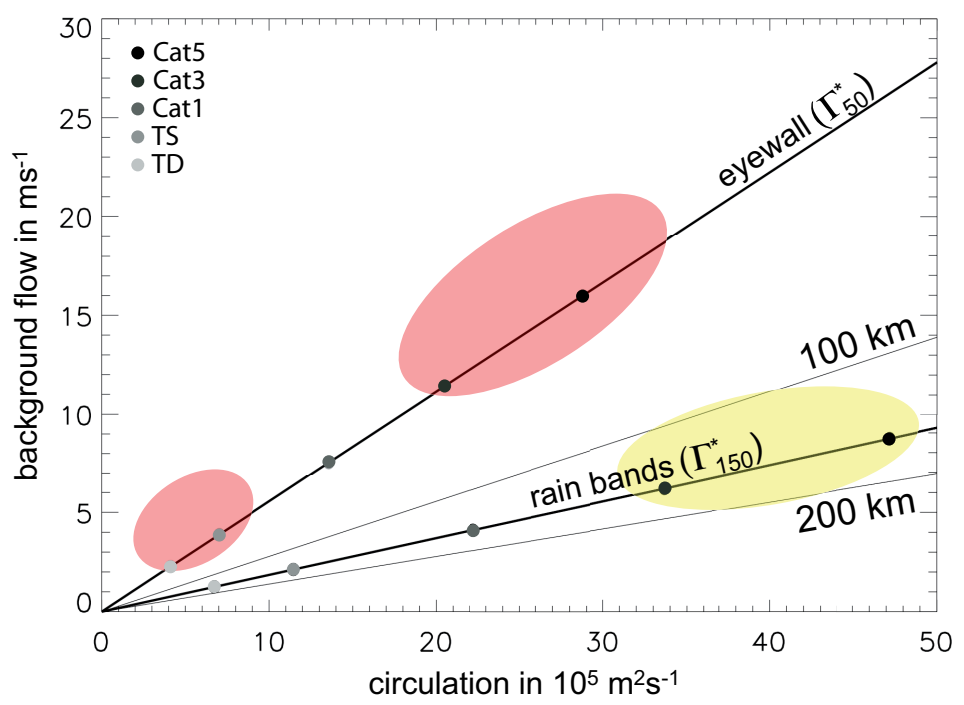

Fig. 8. Regime diagram for the environmental interaction of TCs in vertical wind shear. The sloping lines denote the closest radius of the dividing streamline for a combination of circulation (abscissa) and storm-relative flow (ordinate). For eyewall interaction the TC categories are defined by $\Gamma_{50}$, for rain band interaction $\Gamma_{150}$ is used (see Sect. 3.2.1). The TC categories are highlighted by the shaded dots. The red shading indicates the regime of eyewall interaction for weak and mature TCs (as defined in Table 1), respectively. Yellow shading indicates the regime of rain band interaction for mature TCs. The eyewall radius $(50 \mathrm{~km}), 100 \mathrm{~km}$ radius, the rain band radius $(150 \mathrm{~km})$, and $200 \mathrm{~km}$ radius are depicted (from top to bottom).

less susceptible to easterly than to westerly environmental shear. Future work should test both hypotheses for the potential importance of the shear direction for TC intensity change, e.g. in an idealized modeling framework.

\section{Conclusions}

The flow topology of a numerically simulated, idealized TC in vertical wind shear has been examined under the assumption of steady and layer-wise horizontal flow. Our results show that for an intense TC, the stagnation point and the associated manifolds are found at significantly larger radii than previously hypothesized (WMF84). The layer-wise horizontal flow is weakly convergent above the layer of strong inflow associated with surface friction and below the outflow layer, i.e. approx. between $1.5 \mathrm{~km}-10 \mathrm{~km}$ height. The unstable manifold spirals inwards and ends in the limit cycle, a dividing streamline that encompasses the eyewall in our idealized experiment at all levels. From the perspective of the steady and layer-wise horizontal flow model, the eyewall is well protected from the intrusion of environmental air. Timedependent and/or vertical motions, which are prevalent in the TC inner-core, are necessary to allow intrusion of environmental air into the inner-core convection.

The TC's moist envelope, high- $\theta_{e}$ air that surrounds the eyewall in the inner core, is strongly distorted by the stormrelative environmental flow. At $2 \mathrm{~km}$ height, the moist enve- lope is highly asymmetric with very low $\theta_{e}$ air within $100 \mathrm{~km}$ of the center in the downshear-left quadrant of the storm. In the downshear-right quadrant, high- $\theta_{e}$ air extends to $150 \mathrm{~km}$ radius at this height. The shape of the moist envelope is closely related to the location and shape of the limit cycle, with the highest $\theta_{e}$ air being contained within the limit cycle. We conclude that the distribution of high- and low- $\theta_{e}$ around the TC at low to mid-levels (approx. between $1.5 \mathrm{~km}$ and $5 \mathrm{~km}$ ) is governed in the first approximation by the stirring of convectively modified air by the steady, horizontal flow.

Strong and persistent downdrafts occur where precipitation from the helical updrafts within the stationary band complex falls into relatively dry, low-level environmental air outside of the limit cycle. The steady supply of dry environmental air in this region promotes the persistence and amplitude of the downdraft pattern. In the first $12 \mathrm{~h}$ following the imposition of vertical shear, air from the north and west of the center (downshear and downshear right) feeds into the downdraft region. Subsequently, the downdraft region is fed also by air that wraps around the center from the east and south. Interaction with air from the east and the south, however, is limited to a small radius $(\mathcal{O}(200 \mathrm{~km}))$. This radius is even smaller than indicated by the location of the stable manifold at $\mathcal{O}(300 \mathrm{~km})$. The source region of the downdraft air clearly depends on the direction of the low-level storm-relative environmental flow, and thus on the shear direction. On the basis of the results presented, it stands to reason that in the 

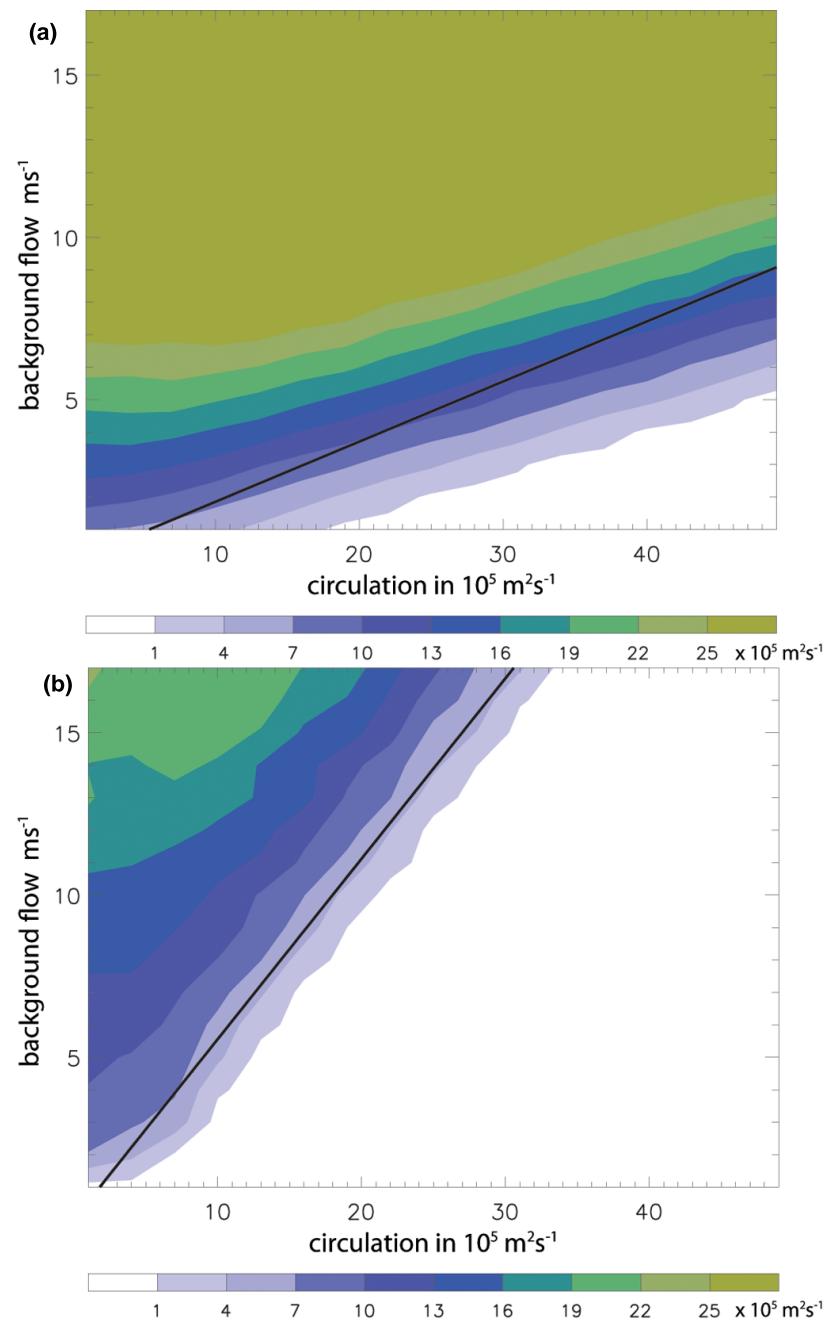

Fig. 9. Inflow rate of environmental air, $\mathcal{F}_{\text {env }}$, (shaded) at the radius of $150 \mathrm{~km}$ indicating rain band interaction (a) and at $50 \mathrm{~km}$ indicating eyewall interaction (b) as a function of circulation $\Gamma$ (abscissa) and storm-relative environmental flow $U$ (ordinate). Values are calculated for the point vortex with centered mass sink $\left(D=-1.5 \times 10^{5} \mathrm{~m}^{2} \mathrm{~s}^{-1}\right)$. The solid line denotes the values of $\Gamma$ and $U$ for which the closest distance of the dividing streamline from the center is at the rain band (a) and eyewall (b) radius, respectively (cf. Fig. 8).

presence of a pronounced environmental moisture gradient, vertical wind shear may thus promote or impede the interaction with dry environmental air, depending on the direction of the shear vector.

To further understand the flow kinematics, an analogue model based on a weakly convergent point vortex in background flow was developed. This simple kinematic model is demonstrated to capture several salient features of the flow topology in the numerical experiment. Assuming a generic distribution of air masses around the TC in a hypothetical "real-world" application, the potential for environmental in-

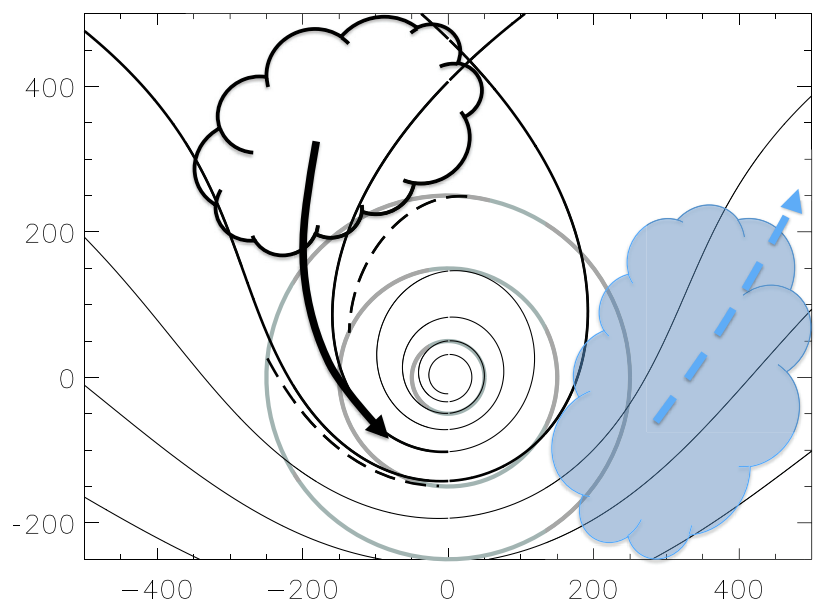

Fig. 10. Same as Fig. 7, but for a Cat 1 vortex in westerly stormrelative environmental flow of $U=5.1 \mathrm{~ms}^{-1}$. The dashed curves denote the envelope of streamlines for which environmental air reaches the "rain band" region in this scenario. The source region of air that interacts with the TC is indicated to the northwest. The area shaded in blue indicates a hypothetical region of very dry environmental air.

teraction can then be quantified by the inflow rate of environmental air to the radius of the principal rain band and eyewall, respectively. The location of the dividing streamline provides a simple, yet adequate approximation for the propensity of environmental interaction. The closest approach of the dividing streamline to the vortex center is proportional to the ratio of the vortex strength and the storm-relative environmental flow. Under the assumption that the low-level stormrelative flow is approximately one half of the difference between the low- and the upper-level flow, distinct scenarios of possible environmental interaction are implicated for the range of point vortices and background flows that represent typical conditions of TCs in vertical wind shear. The results suggest that it is not only the dynamic resiliency of a TC that increases with intensity and vortex size (Jones, 1995; Reasor et al., 2004). The ability of a TC to isolate itself from adverse thermodynamic interaction with dry environmental air increases considerably with intensity and size also.

\section{Appendix A}

\section{Relative importance of $D$ and $U$ for environmental interaction}

The representation of the weakly divergent flow above the TC inflow layer by a singular mass sink is associated with considerable uncertainty (see Sect. 3.2.2). The relative importance of the divergent and storm-relative environmental 
flow in forcing interaction with environmental air is not selfevident. On the one hand, the introduction of a mass sink inside the dividing streamline has a profound impact on the flow topology by opening up a pathway for environmental air to spiral towards the vortex center. On the other hand, the weakly divergent flow is usually much smaller than the storm-relative flow, except very close to the mass sink.

The closest radius of the unstable manifold, $R_{\mathrm{div}}$, is used as a proxy for environmental interaction. We now examine the sensitivity of $R_{\text {div }}$ to small changes in the environmental flow, $U$, and the strength of the mass sink, $D$, respectively:

$\frac{\partial R_{\mathrm{div}}}{\partial U}=-\frac{\alpha}{U^{2}}\left(\Gamma+\frac{\pi}{1+\alpha} D\right)$

and

$$
\frac{\partial R_{\mathrm{div}}}{\partial D}=\frac{\alpha}{U} \frac{\pi}{1+\alpha} .
$$

Dividing Eq. (A1) by Eq. (A2) and using Eqs. (17, 28, and 29) yields

$$
\Delta D=-\frac{1+\alpha}{\pi \alpha} R_{\mathrm{div}} \Delta U
$$

We have replaced the differentials, $\partial U$ and $\partial D$, by small finite changes, $\Delta U$ and $\Delta D$, that lead to the same change in $R_{\text {div }}$.

We may assume a typical variation of the storm-relative environmental flow of $\Delta U=1 \mathrm{~ms}^{-1}$. For storm-relative environmental flow of $4-5 \mathrm{~ms}^{-1}$ this amounts to a relative change of $20-25 \%$. The value of $\Delta D$ that has the same impact on the closest radius of the unstable manifold, $R_{\text {div }}$, depends on $R_{\text {div }}$ itself (Eq. A3). For $R_{\text {div }}=50 \mathrm{~km}$ and $R_{\text {div }}=150 \mathrm{~km}$ we find values of $\Delta D=-0.7 \times 10^{5} \mathrm{~m}^{2} \mathrm{~s}^{-1}$ and $\Delta D=-2.2 \times 10^{5} \mathrm{~m}^{2} \mathrm{~s}^{-1}$, respectively. For the standard value of $D=-1.5 \times 10^{5} \mathrm{~m}^{2} \mathrm{~s}^{-1}$ these values correspond to a relative change of $\sim 50 \%$ and $\sim 150 \%$, respectively. The required relative change of $D$ decreases as $R_{\text {div }}$ decreases, consistent with the increase of the divergent radial wind with decreasing radius (Eq. 6).

It is clear that considerably larger relative changes of $D$ than of $U$ are needed to have the same impact on $R_{\text {div }}$. Given that $R_{\text {div }}$ provides a proxy for environmental interaction, it follows that changes in the storm-relative environmental flow rather than changes of the divergent flow associated with the TC govern the propensity for TC-environment interaction. The general features of our results should thus hold true even in the light of the considerable uncertainty associated with the representation of the weakly divergent flow.

Considering $\mathcal{F}_{\text {env }}$ over a range of values of $U$ and $D$ for specified $\Gamma$ (not shown) confirms that the variation of $R_{\mathrm{div}}$ with $U$ and $D$ is indeed a good approximation for the relative importance of the weakly divergent and the storm-relative environmental flow. However, when the outermost streamline that connects the environmental radius with the inner core (southern dashed line in Fig. 10) orbits the center before reaching the inner core, $\mathcal{F}_{\text {env }}$ is constrained by $D$ alone. For these slowly inward spiraling streamlines, however, our assumptions of steady and layer-wise horizontal flow are likely to break down, as discussed in Sect. 4.2.

Acknowledgements. This research was performed while the first author held a National Research Council Research Associateship Award at the Naval Postgraduate School. We acknowledge helpful discussions with Kerry Emanuel and Scott Braun. We thank also Michael Bell and Blake Rutherford for their insightful comments on an earlier version of this manuscript. The reviews of two anonymous referees and of Brian Tang helped to improve the presentation of our results. This work is supported by NASA grant NNG09HG031, NFS grant ATM-0649946, NSF Cooperative Agreement ATM-0715426, and ONR grant N000014-03-1-0185.

Edited by: J. Dunkerton

\section{References}

Barnes, G. M., Zipser, E. J., Jorgensen, D., and Marks, F.: Mesoscale and convective structure of a hurricane rainband, J. Atmos. Sci., 40, 2125-2137, 1983.

Bender, M. A.: The effect of relative flow on the asymmetric structure in the interior of hurricanes, J. Atmos. Sci., 54, 703-724, 1997.

Black, M. L., Gamache, J. F., Marks, F. D., Samsury, C. E., and Willoughby, H. E.: Eastern Pacific Hurricanes Jimena of 1991 and Olivia of 1994: The effect of vertical shear on structure and intensity, Mon. Weather Rev., 130, 2291-2312, 2002.

Bolton, D.: The computation of equivalent potential temperature, Mon. Weather Rev., 108, 1046-1053, 1980.

Bui, H. H., Smith, R. K., Montgomery, M. T., and Peng, J.: Balanced and unbalanced aspects of tropical-cyclone intensification, Q. J. R. Meteorol. Soc., 135, 715-1731, 2009.

Carr, L. E. and Williams, R. T.: Barotropic vortex stability to perturbations from axisymmetry, J. Atmos. Sci., 46, 3177-3191, 1989.

Charney, J. G.: A note on large-scale motions in the tropics, J. Atmos. Sci., 20, 607-609, 1963.

Chen, Y. and Yau, M. K.: Spiral bands in a simulated hurricane. Part I: Vortex Rossby wave verification, J. Atmos. Sci., 58, 2128 2145, 2001.

Chen, Y., Brunet, G., and Yau, M. K.: Spiral bands in a simulated hurricane. Part II: Wave Activity Diagnostics, J. Atmos. Sci., 60, 1240-1256, 2003.

Cotton, W. R., Pielke, R. A., Walko, R. L., Liston, G. E., Tremback, C. J., Jiang, H., McAnelly, R. L., Harrington, J. Y., Nicholls, M. E., Carrio, G. G., and McFadden, J. P.: RAMS 2001: Current status and future directions, Meteorol. Atmos. Phys., 82, 5-29, 2003.

Cram, T. A., Persing, J., Montgomery, M. T., and Braun, S. A.: A Lagrangian trajectory view on transport and mixing processes between the eye, eyewall, and environment using a highresolution simulation of Hurricane Bonnie (1998), J. Atmos. Sci., 64, 1835-1856, 2007.

Dunion, J. P. and Velden, C. S.: The impact of the Saharan air layer on Atlantic tropical cyclone activity, B. Am. Meteorol. Soc., 85, 353-365, 2004. 
Dunkerton, T. J., Montgomery, M. T., and Wang, Z.: Tropical cyclogenesis in a tropical wave critical layer: easterly waves, Atmos. Chem. Phys., 9, 5587-5646, doi:10.5194/acp-9-5587-2009, 2009.

Emanuel, K., DesAutels, C., Holloway, C., and Korty, R.: Environmental control of tropical cyclone intensity, J. Atmos. Sci., 61, 843-858, 2004.

Frank, W. M. and Ritchie, E. A.: Effects of vertical wind shear on the intensity and structure of numerically simulated hurricanes, Mon. Weather Rev., 129, 2249-2269, 2001.

Haller, G.: Distinguished material surfaces and coherent structures in three-dimensional fluid flows, Physica D, 149, 248-277, 2001.

Holton, J.: An introduction to dynamic meteorology, AcademicPress, London, 4th edn., 387-389, 2004.

Houze, R. A., Cetrone, J., Brodzik, S. R., Chen, S. S., Zhao, W., Lee, W.-C., Moore, J. A., Stossmeister, G. J., Bell, M. M., and Rogers, R. F.: The hurricane rainband and intensity change experiment: Observations and modeling of Hurricanes Katrina, Ophelia, and Rita, B. Am. Meteorol. Soc., 87, 1503-1521, 2006.

Ide, K., Small, D., and Wiggins, S.: Distinguished hyperbolic trajectories in time-dependent fluid flows: Analytical and computational approach for velocity fields defined as data sets, Nonlinear Proc. Geoph., 9, 237-263, 2002.

Jones, S. C.: The evolution of vortices in vertical shear. I: Initially barotropic vortices, Q. J. R. Meteorol. Soc., 121, 821-851, 1995.

Kimball, S. K.: A modeling study of hurricane landfall in a dry environment, Mon. Weather Rev., 134, 1901-1918, 2006.

Lamb, H.: Hydrodynamics, Dover Publications, New York, 6th edn., 1945.

Mallen, K. J., Montgomery, M. T., and Wang, B.: Reexamining the near-core radial structure of the tropical cyclone primary circulation: implications for vortex resiliency, J. Atmos. Sci., 62, 408-425, 2005.

Marks, F. D., Houze, R. A., and Gamache, J. F.: Dual-aircraft investigation of the inner core of Hurricane Norbert. Part I: Kinematic structure, J. Atmos. Sci., 49, 919-942, 1992.

Melander, M. V., McWilliams, J. C., and Zabusky, N. J.: Axisymmetrization and vorticity-gradient intensification of an isolated two-dimensional vortex through filamentation, J. Fluid Mech., 178, 137-159, 1987.

Montgomery, M. T. and Kallenbach, R. J.: A theory for vortex Rossby-waves and its application to spiral bands and intensity changes in hurricanes, Q. J. R. Meteorol. Soc., 123, 435-465, 1997.

Nguyen, S. V., Smith, R. K., and Montgomery, M. T.: Tropicalcyclone intensification and predictability in three dimensions, $\mathrm{Q}$. J. R. Meteorol. Soc., 134, 563-582, 2008.

Pielke, R. A., Cotton, W. R., Walko, R. L., Tremback, C. J., Lyons, W. A., Grasso, L. D., Nicholls, M. E., Moran, M. D., Wesley, D. A., Lee, T. J., and Copeland, J. H.: A comprehensive meteorological modeling system - RAMS, Meteorol. Atmos. Phys., 49, 69-91, 1992.

Powell, M. D.: Boundary layer structure and dynamics in outer hurricane rainbands. Part II: Downdraft modification and mixed layer recovery, Mon. Weather. Rev., 118, 918-938, 1990.

Reasor, P. D., Montgomery, M. T., and Grasso, L. D.: A new look at the problem of tropical cyclones in vertical shear flow: Vortex resiliency, J. Atmos. Sci., 61, 3-22, 2004.
Riemer, M., Montgomery, M. T., and Nicholls, M. E.: A new paradigm for intensity modification of tropical cyclones: thermodynamic impact of vertical wind shear on the inflow layer, Atmos. Chem. Phys., 10, 3163-3188, doi:10.5194/acp-10-31632010, 2010.

Ritchie, E. A. and Frank, W. M.: Interactions between simulated tropical cyclones and an environment with a variable Coriolis parameter, Mon. Weather Rev., 135, 1889-1905, 2007.

Roy, R. and Olver, F. W. J.: Lambert W function, in: NIST Handbook of Mathematical Functions, edited by Olver, F. W. J., Lozier, D. M., Boisvert, R. F., and Clark, C. W., Cambridge University Press, 2010.

Rutherford, B., Dangelmayr, G., and Montgomery, M. T.: Lagrangian Coherent Structures in tropical cyclone intensification, Atmos. Chem. Phys. Disc., accepted, 2010.

Sapsis, T. and Haller, G.: Inertial particle dynamics in a hurricane, J. Atmos. Sci., 66, 2481-2492, 2009.

Shelton, K. L. and Molinari, J.: Life of a six-hour hurricane, Mon. Weather Rev., 137, 51-67, 2009.

Simpson, R. H. and Riehl, H.: Mid-tropospheric ventilation as a constraint on hurricane development and maintenance, in: Proc. Tech. Conf. on Hurricanes, D4.1-D4.10, Amer. Meteorol. Soc., Miami, FL, 1958.

Smith, R. K., Ulrich, W., and Sneddon, G.: On the dynamics of hurricane-like vortices in vertical-shear flows, Q. J. R. Meteorol. Soc., 126, 2653-2670, 2000.

Tang, B. and Emanuel, K. A.: Midlevel ventilation's constraint on tropical cyclone intensity, J. Atmos. Sci., 67, 1817-1830, 2010a.

Tang, B. and Emanuel, K. A.: Entropy ventilation in an axisymmetric tropical cyclone model, in: 29th Conference on Hurricanes and Tropical Meteorology, 7C.2, Amer. Meteorol. Soc., Tucson, AZ, 2010b.

Wang, Y.: Vortex Rossby waves in a numerically simulated tropical cyclone. Part I: Overall structure, potential vorticity, and kinetic energy budgets, J. Atmos. Sci., 59, 1213-1238, 2002.

Weatherford, C. L. and Gray, W. M.: Typhoon structure as revealed by aircraft reconnaissance. Part II: Structural variability, Mon. Weather Rev., 116, 1044-1056, 1988.

Willoughby, H. E., Marks, F. D., and Feinberg, R. J.: Stationary and moving convective bands in hurricanes, J. Atmos. Sci., 41, 3189-3211, 1984.

Zehr, R. M.: Environmental vertical wind shear with Hurricane Bertha (1996), Weather Forecast., 18, 345-356, 2003.

Zhang, D.-L. and Kieu, C. Q.: Potential vorticity diagnosis of a simulated hurricane. Part II: Quasi-balanced contributions to forced secondary circulations, J. Atmos. Sci., 63, 2898-2914, 2006.

Zipser, E. J., Twohy, C. H., Tsay, S.-C., Thornhill, K. L., Tanelli, S., Ross, R., Krishnamurti, T. N., Ji, Q., Jenkins, G., Ismail, S., Hsu, N. C., Hood, R., Heymsfield, G. M., Heymsfield, A., Halverson, J., Goodman, H. M., Ferrare, R., Dunion, J. P., Douglas, M., Cifelli, R., Chen, G., Browell, E. V., and Anderson, B.: The Saharan air layer and the fate of African easterly waves: NASA's AMMA field study of tropical cyclogenesis, B. Am. Meteorol. Soc., 90, 1137-1156, 2009. 